\title{
X-ray and optical spectroscopy of the massive young open cluster IC $1805^{\star, \star \star}$
}

\author{
G. Rauw and Y. Nazé ${ }^{\star \star \star}$ \\ Space sciences, Technologies and Astrophysics Research (STAR) Institute, Université de Liège, Allée du 6 Août, 19c, Bât. B5c,
4000 Liège, Belgium \\ e-mail: rauw@astro.ulg.ac.be
}

Received 29 June 2016 / Accepted 18 July 2016

\begin{abstract}
Context. Very young open clusters are ideal places to study the X-ray properties of a homogeneous population of early-type stars. In this respect, the IC 1805 open cluster is very interesting as it hosts the $\mathrm{O}_{4} \mathrm{If}^{+}$star HD 15570 thought to be in an evolutionary stage intermediate between a normal O-star and a Wolf-Rayet star.

Aims. Such a star could provide a test for theoretical models aiming at explaining the empirical scaling relation between the X-ray and bolometric luminosities of O-type stars.

Methods. We have observed IC 1805 with XMM-Newton and further collected optical spectroscopy of some of the O-star members of the cluster.

Results. The optical spectra allow us to revisit the orbital solutions of BD+60 497 and HD 15558, and provide the first evidence of binarity for $\mathrm{BD}+60^{\circ} 498$. X-ray emission from colliding winds does not appear to play an important role among the O-stars of IC 1805. Notably, the X-ray fluxes do not vary significantly between archival X-ray observations and our XMM-Newton pointing. The very fast rotator $\mathrm{BD}+60^{\circ} 513$, and to a lesser extent the $\mathrm{O} 4 \mathrm{If}^{+}$star HD 15570 appear somewhat underluminous. Whilst the underluminosity of HD 15570 is only marginally significant, its amplitude is found to be compatible with theoretical expectations based on its stellar and wind properties. A number of other X-ray sources are detected in the field, and the brightest objects, many of which are likely low-mass pre-main sequence stars, are analyzed in detail.
\end{abstract}

Key words. stars: early-type - open clusters and associations: individual: IC 1805 - X-rays: stars

\section{Introduction}

X-ray emission of massive O-type stars was discovered in the late seventies with the Einstein satellite (Harnden et al. 1979). $\mathrm{X}$-rays in single $\mathrm{O}$-stars are generally thought to arise from a distribution of hydrodynamic shocks produced by the so-called line deshadowing instability (LDI, e.g. Feldmeier et al. 1997) that affects the radiatively driven winds of these objects. Additional $\mathrm{X}$-ray emission can arise in magnetically confined stellar winds of O-stars with a strong enough magnetic field (e.g. Babel \& Montmerle 1997; ud-Doula \& Owocki 2002; Nazé et al. 2014; ud-Doula \& Nazé 2016, and references therein), or in interacting wind binary systems (e.g. Stevens et al. 1992; Pittard \& Parkin 2010; Rauw \& Nazé 2016, and references therein).

Soon after the discovery of the X-ray emission of O-type stars with the Einstein satellite, it was realised that their X-ray luminosity $L_{\mathrm{X}}$ scales with their bolometric luminosity $L_{\mathrm{bol}}$ (e.g. Sciortino et al. 1990). This scaling relation was confirmed and refined with large samples of O-type stars observed with ROSAT (Berghöfer et al. 1997), and most recently XMM-Newton

\footnotetext{
* Based on observations collected with XMM-Newton, an ESA science mission with instruments and contributions directly funded by ESA member states and the USA (NASA), and with the TIGRE telescope (La Luz, Mexico).

$\star \star$ Table A. 1 is only available at the CDS via anonymous ftp to cdsarc.u-strasbg.fr (130.79.128.5) or via

http://cdsarc.u-strasbg.fr/viz-bin/qcat?J/A+A/594/A82

$\star \star \star$ Research Associate FRS-FNRS (Belgium).
}

(Sana et al. 2006b; Nazé 2009) and Chandra (Nazé et al. 2011; Rauw et al. 2015). A very different situation holds for presumably single Wolf-Rayet stars, where no clear dependence between X-ray and bolometric luminosities exists (Wessolowski 1996; Ignace et al. 2000; Oskinova 2015). Some classes of WolfRayet stars remain below the detection threshold of the current generation of X-ray observatories (e.g. Oskinova et al. 2003; Gosset et al. 2005).

Owocki et al. (2013) attempted to theoretically explain the origin of the empirical $L_{\mathrm{X}} / L_{\mathrm{bol}}$ scaling relation of O-stars. They predicted that X-ray emission produced by LDI shocks should scale as $L_{\mathrm{X}} \propto L_{\mathrm{bol}}^{1.7}$ or $L_{\mathrm{X}} \propto L_{\mathrm{bol}}^{3.4}$ for radiative and adiabatic shocks, respectively. These authors also argued that the shocks in the winds of most O-star are most likely radiative, although the wind density remains sufficiently low in most cases to prevent the wind absorption from playing a significant role. Owocki et al. (2013) considered that turbulence in the radiatively cooling postshock gas efficiently mixes cold and hot material. To reproduce the observed linear $L_{\mathrm{X}}$ versus $L_{\mathrm{bol}}$ relation, these authors adopted a scaling of the hot gas volume filling factor with some ad hoc power $m \simeq 0.2-0.4$ of the ratio between cooling length and position in the wind. Following their formalism, the X-ray luminosity should scale as $\left(\frac{\dot{M}}{v_{\infty}}\right)^{1-m}$ (where $\dot{M}$ and $v_{\infty}$ are the mass-loss rate and the terminal velocity of the stellar wind) over most of the spectral range of O-type stars, where the LDI shocks that produce the X-rays are radiative and the winds themselves are optically thin. Such a relation between the X-ray flux (corrected 
for the absorption of the interstellar medium, ISM) and the theoretical value of $\frac{\dot{M}}{v_{\infty}}$ was indeed found for the O-type stars observed within the Chandra Cyg OB2 legacy survey (Rauw et al. 2015). The Owocki et al. (2013) scenario furthermore predicts a change in the behaviour of the $L_{\mathrm{X}}$ versus $L_{\mathrm{bol}}$ relation at the highluminosity, high mass-loss rate end of the O-star domain, where the winds should progressively become optically thick. Gayley (2016) extended the Owocki et al. (2013) formalism and proposed a first unified approach to estimate the X-ray emergence efficiency for winds of non-magnetic hot stars. He predicted a smooth evolution of the X-ray emergence efficiency over the full range of wind strengths from early B-stars into Wolf-Rayet stars.

The most extreme O-stars are so-called $\mathrm{O} \mathrm{If}^{+}$stars. These objects have spectral properties intermediate between those of normal O-stars and those of WN-type Wolf-Rayet stars (Willis \& Stickland 1980; Conti et al. 1995; De Becker et al. 2009) and are therefore considered to be transition objects. Possible indications for a difference in the $L_{\mathrm{X}} / L_{\mathrm{bol}}$ ratio for $\mathrm{O} \mathrm{If}^{+}$stars were reported by De Becker (2013), who found for the O4 $\mathrm{If}^{+}$ and $05 \mathrm{f}^{+}$stars HD 16691 and HD $14947 \log L_{\mathrm{X}} / L_{\text {bol }} \sim-7.1$ or -7.5 depending on the adopted value of their $L_{\text {bol }}$. However, because these two stars are rather isolated, that is, are located outside a stellar cluster, a proper $L_{\mathrm{X}} / L_{\text {bol }}$ benchmark is lacking against which a genuine X-ray underluminosity might be established. Whilst the X-ray emissions of O-type stars inside a specific cluster exhibit only a very small scatter about the mean $L_{\mathrm{X}} / L_{\text {bol }}$ ratio, values ranging between -6.5 and -7.2 have been reported for $\log L_{\mathrm{X}} / L_{\mathrm{bol}}$ in different populations. Some of the dispersion around the $L_{\mathrm{X}} / L_{\text {bol }}$ relation inferred from large samples of O-stars (mixing objects from different clusters and field stars) may be due to environmental effects, different approaches in the data analysis (Nazé 2009; Nazé et al. 2011), or even a poor determination of the spectral properties when the interstellar absorption is high (Nazé et al. 2013a; Mernier \& Rauw 2013). Therefore, an X-ray over- or underluminosity is hard to demonstrate for an isolated $\mathrm{O}$-star.

The best way to probe the $L_{\mathrm{X}} / L_{\mathrm{bol}}$ ratio in the higher luminosity regime, is to observe a cluster hosting an $\mathrm{O} \mathrm{If}^{+}$star, along with a significant population of normal O-stars, which allows homogeneously building the local $L_{\mathrm{X}} / L_{\mathrm{bol}}$ relation of normal O-stars. The only Galactic young open cluster hosting a large sample of O-stars and including an $\mathrm{O}_{4} \mathrm{If}^{+}$star (HD 15570) is IC 1805 at a distance near $2 \mathrm{kpc}$ (Megeath et al. 2008).

Within a radius of $12^{\prime}$ around the central $\mathrm{O}+\mathrm{O}$ binary HD 15558, this cluster hosts about 40 early-type stars in the spectral range $\mathrm{O} 4-\mathrm{B} 2$, including 8 O-stars with spectral types from O9.5 V to O4 If $^{+}$(Massey et al. 1995; Shi \& Hu 1999). IC 1805 is not only an interesting place to study the X-ray properties of the population of massive stars, but is also an important site to study star formation. IC 1805 lies at the centre of a large superbubble inside the W4 region (Megeath et al. 2008, and references therein). The massive stars have created an $\mathrm{H}$ II region that is surrounded by a cloud of molecular (CO) and atomic (H I) gas. The cluster is embedded in a bubble of hot plasma produced by the winds of the O-stars, which yields a diffuse X-ray emission detected with Chandra (Townsley et al. 2014). IC 1805 and the entire W4 complex have been the theatre of multiple episodes of star formation over the past 10-20 Myr and low-mass star formation is still ongoing (Megeath et al. 2008; Straižys et al. 2013; Sung et al., in prep.), although photoevaporation by the harsh UV-radiation fields of the massive cluster members led to the destruction of optically thick accretion disks around most of the intermediate mass stars (Wolff et al. 2011).

In this paper we discuss a new X-ray observation of the massive star population of IC 1805 along with some support optical spectroscopy. Our observational material is described in Sect. 2. The results of our optical monitoring are provided in Sect. 3, and our X-ray data are analysed and discussed in Sect. 4. The summary of our results is given in Sect. 5 .

\section{Observations}

\subsection{XMM-Newton}

XMM-Newton (Jansen et al. 2001) observed IC 1805 for $49 \mathrm{ks}$ in August 2014 (PI Rauw, ObsID 0740020101, see Table 1). The raw data were reduced with SAS v14.0.0 using calibration files available in November 2015 and following the recommendations of the XMM-Newton team ${ }^{1}$. The EPIC (Strüder et al. 2001; Turner et al. 2001) cameras were operated in the fullframe mode and the medium filter was used to reject optical and UV light. During reduction, these data were filtered to keep only best-quality data (PATTERN of 0-12 for EPIC-MOS and 0-4 for EPIC-pn data). We note that no background flares due to soft protons affected the observation.

Source detection was performed on the three EPIC datasets using the task edetect_chain on the 0.4-2.0 (soft) and 2.0-10.0 (hard) energy bands for a log-likelihood of 10. This task searches for sources using a sliding box and determines the final source parameters from point spread function (PSF) fitting. The final count rates correspond to equivalent on-axis, full PSF count rates. The task was run both with and without considering the possibility of extended sources and simultaneous fit of up to five neighbouring sources. The results were similar in both cases. A total of 191 sources were found, nine of them appearing potentially problematic (e.g. because of their position in or near a CCD gap, or in the wings of the PSF of a brighter source). The full catalogue of sources is given in Table A.1. The properties of the brightest X-ray sources are analysed in Sect. 4, whilst a full correlation with optical catalogues is performed in Sung et al. (in prep.).

We then extracted spectra using the task especget for the eight detected massive stars and for 17 other sources having at least 400 EPIC counts. For the source regions, we used circular regions with radii between 7 and $25^{\prime \prime}$ (depending on crowding in the area) and centred on the Simbad coordinates of the massive stars or on the best-fit positions found in the source detection step for the other sources. For the background regions, circular regions of at least $18^{\prime \prime}$ radius were chosen in a region devoid of sources and as close as possible to the targets. Dedicated ARF and RMF responses, which are used to calibrate the flux and energy axes, respectively, were also calculated. The EPIC spectra were grouped with the SAS command specgroup to obtain an oversampling factor of five and to ensure that at least a signalto-noise ratio of 3 (i.e. a minimum of ten counts) was reached in each spectral bin of the background-corrected spectra.

EPIC light curves of the brightest sources were extracted for time bins of $1 \mathrm{ks}$ and for the total $0.4-10 . \mathrm{keV}$ energy band in the same regions as the spectra. They were further processed by the task epiclccorr, which corrects for loss of photons due to vignetting, off-axis angle, or other problems such as bad pixels. In addition, to avoid very large errors and poor estimates

\footnotetext{
http://xmm.esac.esa.int/sas/current/documentation/
} threads/ 
Table 1. Journal of X-ray observations of IC 1805.

\begin{tabular}{ccccc}
\hline \hline Observatory & Inst. & $\begin{array}{c}\text { Duration } \\
(\mathrm{ks})\end{array}$ & $\begin{array}{c}\mathrm{JD}(\mathrm{start}) \\
-2440000\end{array}$ & $\begin{array}{c}\mathrm{JD}(\mathrm{end}) \\
-2440000\end{array}$ \\
\hline ROSAT & PSPC-B & 8.5 & 8856.966 & 8858.827 \\
Chandra & ACIS-I & 82.1 & 14065.465 & 14066.416 \\
XMM-Newton & EPIC & 48.7 & 16895.380 & 16895.943 \\
\hline
\end{tabular}

of the count rates, we discarded bins displaying effective exposure times lower than $50 \%$ of the time bin length. Our previous experience with $X M M$-Newton has shown us that including such bins degrades the results. As the background is much fainter than the source, in fact too faint to provide a meaningful analysis, three sets of light curves were produced and analysed individually: the raw source+background light curves, the backgroundcorrected light curves of the source, and finally the light curves of the background region alone. The results found for the raw and background-corrected light curves of the source are indistinguishable. As for $\zeta$ Pup (Nazé et al. 2013b), the same set of tests was applied to all cases. We first performed a $\chi^{2}$ test for three different null hypotheses (constancy, linear variation, quadratic variation), and also compared the improvement of the $\chi^{2}$ when increasing the number of parameters in the model (e.g. linear trend versus constancy) by means of Snedecor F tests (nested models, see Sect. 12.2.5 in Lindgren 1976).

\subsection{Archival ROSAT and Chandra data}

IC 1805 was also observed during $80 \mathrm{ks}$ with Chandra in November 2006 (PI Townsley, ObsID 7033, see Table 1, Feigelson et al. 2013; Townsley et al. 2014). We processed these data using the CIAO v.4.7 software (with CALDB 4.6.9). Using specextract, we extracted the spectra and generated sourcespecific response matrices for six massive stars $\left(\mathrm{BD}+60^{\circ} 498\right.$, $\mathrm{BD}+60^{\circ} 499, \mathrm{BD}+60^{\circ} 501$, HD $15558^{2}$, HD 15570, and HD 15629) as well as six bright XMM-Newton sources (see Sect. 4.3) with at least 100 net ACIS counts as reported in Townsley et al. (2014). To this aim, we used circular regions of radius between 2 and 15", depending on the off-axis angle and crowding ${ }^{3}$. Circular background regions as close as possible to the targets were used and a similar grouping as for the $X M M-N e w t o n$ data was applied.

Finally, we also retrieved an archival ROSAT observation (PI Pauldrach, ObsID 201263, see Table 1) obtained in August 1992. These data were processed using the xselect software. Spectra for five O-stars (BD+60 497, BD+60 501, HD 15558, HD 15570, and HD 15629) were extracted using circular extraction regions of radius $42^{\prime \prime}$. The background was evaluated over a circular source-free region of $75^{\prime \prime}$ radius. We used the standard response matrix pspcb_gain2_256.rmf and generated source-specific ancillary response files using the pcarf command.

\footnotetext{
2 The ACIS spectrum of this star is subject to moderate pile-up, see Townsley et al. (2014).

3 To evaluate crowding, we used the source list of Townsley et al. (2014). BD $+60^{\circ} 499$ appears to have a close companion, which is blended with the O-star emission in XMM-Newton data, therefore we extracted the spectra of $\mathrm{BD}+60^{\circ} 499$ and its companion to evaluate the contamination in the XMM-Newton data. The small separation of the two sources forced the use of ellipses, rather than circles, to extract their spectra.
}

\subsection{Optical spectroscopy}

In support of the XMM-Newton observation, we collected optical spectroscopy for several O-type stars of IC 1805 with the refurbished HEROS spectrograph at the $1.2 \mathrm{~m}$ TIGRE telescope (Schmitt et al. 2014) at La Luz Observatory (Guanajuato, Mexico). The HEROS echelle spectra have a resolving power of 20000 and cover the full optical range, although with a small gap near $5800 \AA$. The HEROS data were reduced with the corresponding reduction pipeline (Mittag 2010; Schmitt et al. 2014). Telluric absorptions in the $\mathrm{He} \mathrm{I} \lambda 5876$ and $\mathrm{H} \alpha$ regions were corrected with the telluric command within IRAF using the list of telluric lines of Hinkle et al. (2000). The spectra were normalized self-consistently using a series of carefully chosen continuum windows. Journals of the observations for each star, along with the radial velocities that we have determined are given in Tables B.1-B.7.

\section{Optical monitoring of the O-stars in IC $\mathbf{1 8 0 5}$}

To assess the $L_{\mathrm{X}} / L_{\mathrm{bol}}$ relation of single O-type stars in IC 1805 , it is important to derive accurate spectral types and evaluate the contamination of our sample by colliding wind binaries. So far, investigations of the multiplicity of O-type stars in IC 1805 only revealed two binaries: the long-period binary HD 15558 $\left(\mathrm{O} 5.5 \mathrm{III}(\mathrm{f})+\mathrm{O} 7 \mathrm{~V}, P_{\text {orb }}=442\right.$ days, $e=0.4$; Garmany \& Massey 1981; De Becker et al. 2006), and the short-period system BD+60 $497\left(\mathrm{O} 6.5 \mathrm{~V}((\mathrm{f}))+\right.$ O8.5-9.5 V((f)), $P_{\text {orb }}=$ 3.96 days; Rauw \& De Becker 2004). In this section, we briefly review the properties of the O-star population within the field of view of our X-ray observations and, whenever appropriate, we revise them using our HEROS spectra.

Membership of the stars in the field of IC 1805 was investigated by Vasilevskis et al. (1965) based on proper motion. The corresponding membership probabilities were subsequently revised by Sanders (1972). Except for HD 15558, all O-stars considered here have a membership probability exceeding 50\% (Sanders 1972). For some of the stars, proper motion membership probabilities were also derived from HIPPARCos data (Baumgardt et al. 2000). Unfortunately, for the few stars in common between the studies of Sanders (1972) and Baumgardt et al. (2000), the results often differ significantly ${ }^{4}$. Nevertheless, in what follows, we assume that all the O-type stars belong to IC 1805.

\section{1. $H D 15558$}

The spectrum of HD 15558 displays a number of remarkable features (see discussion by De Becker et al. 2006). The combined spectral type of the system was recently given as O4.5 III(f) by Sota et al. (2011). De Becker et al. (2006) tentatively proposed an O5.5 III(f) + O7 V spectral classification for the binary system.

Garmany \& Massey (1981) presented the first single-lined spectroscopic binary (SB1) orbital solution of this eccentric long-period ( $\sim 40$ days) spectroscopic binary system. The SB1 orbital parameters were improved by De Becker et al. (2006). These authors also identified weak spectroscopic signatures of the secondary star in some spectral lines, which led to a first double-lined spectroscopic binary (SB2) orbital solution. The latter yielded very high minimum masses for the primary star

\footnotetext{
4 For instance, HD 15558 has a cluster membership probability of $2 \%$ according to Sanders (1972) and of $94.8 \%$ according to Baumgardt et al. (2000).
} 
Table 2. Revised SB1 orbital solution of HD 15558 based on RVs of the He II $\lambda 4542$ line (see Table B.1).

\begin{tabular}{cc}
\hline \hline Element & Value \\
\hline$P_{\text {orb }}($ days $)$ & $445.76 \pm 0.42$ \\
$e$ & $0.42 \pm 0.02$ \\
$T_{0}(\mathrm{HJD})$ & $2456692.47 \pm 3.71$ \\
$K\left(\mathrm{~km} \mathrm{~s}^{-1}\right)$ & $38.7 \pm 1.1$ \\
$\gamma\left(\mathrm{km} \mathrm{s}^{-1}\right)$ & $-40.7 \pm 0.8$ \\
$\omega\left(^{\circ}\right)$ & $120.2 \pm 4.3$ \\
$a \sin i\left(R_{\odot}\right)$ & $309.4 \pm 9.8$ \\
$f(m)\left(M_{\odot}\right)$ & $2.00 \pm 0.19$ \\
\hline
\end{tabular}

$\left(m_{1} \sin ^{3} i>150 M_{\odot}\right)$. However, this result needs to be confirmed, as the luminosities of HD 15558 (assuming the star to be a member of IC 1805) would be surprisingly low for such a massive star. De Becker et al. (2006) suggested that HD 15558 might be a triple system with a short-period binary moving around the O5 III star in a wide orbit of $\sim 440$ days.

We have analysed 16 new HEROS spectra of HD 15558 taken between August 2013 and October 2014. Whilst this dataset is not sufficient to search for a possible signature of the putative close binary system, it allows us to improve the SB1 orbital solution and to establish accurate orbital phases corresponding to the X-ray observations. We measured the radial velocities (RVs) of the He II $\lambda 4542$ line on our HEROS spectra (Table B.1) and combined these new RVs with those listed by De Becker et al. (2006). Using the Fourier period search technique of Heck et al. (1985) and Gosset et al. (2001), we found a best estimate of the orbital period of $447.25 \pm 4.10$ days for $87 \mathrm{RV}$ data points spread over 4880 days. We then used the Liège Orbital Solution Package (LOSP) code (Sana et al. 2006a), which is an improved version of the code originally proposed by Wolfe et al. (1967). The resulting orbital elements are listed in Table 2 and the new orbital solution is shown in Fig. 1. We note that the new mass function is lower than in the solution of De Becker et al. (2006), which obviously also leads to a reduction of the masses of the individual components. More observations are needed to determine whether HD 15558 is a triple system and to search for the secondary (and possibly tertiary) spectral signatures.

As a massive binary system, HD 15558 hosts a colliding wind interaction. The star was reported to be a probable nonthermal radio emitter by Bieging et al. (1989), and the relativistic electrons involved in the synchrotron radio emission are most likely accelerated at the shock fronts of the wind - wind collision. The wind interaction zone is also most relevant in the context of the X-ray emission of the system. With the newly derived ephemerides, we find that the ROSAT, Chandra and XMM-Newton observations were taken at orbital phases 0.42 , 0.11 and 0.46 , respectively, where $\phi=0.0$ corresponds to the periastron passage.

\section{2. $H D 15570$}

The optical spectrum of HD 15570 displays many peculiarities (notably strong, broad, and asymmetric $\mathrm{H} \alpha$ emission, presence of Si IV $\lambda \lambda$ 4088, 4116 and weak N IV $\lambda 4058$ emissions in addition to the usual Of emission lines of He II $\lambda 4686$ and N III $\lambda \lambda 4634-4640$, presence of $\mathrm{NV} \lambda \lambda 4604,4620$ absorptions) that led to an $\mathrm{O}_{4} \mathrm{If}^{+}$classification. A full discussion of the optical spectrum is provided by De Becker et al. (2006).

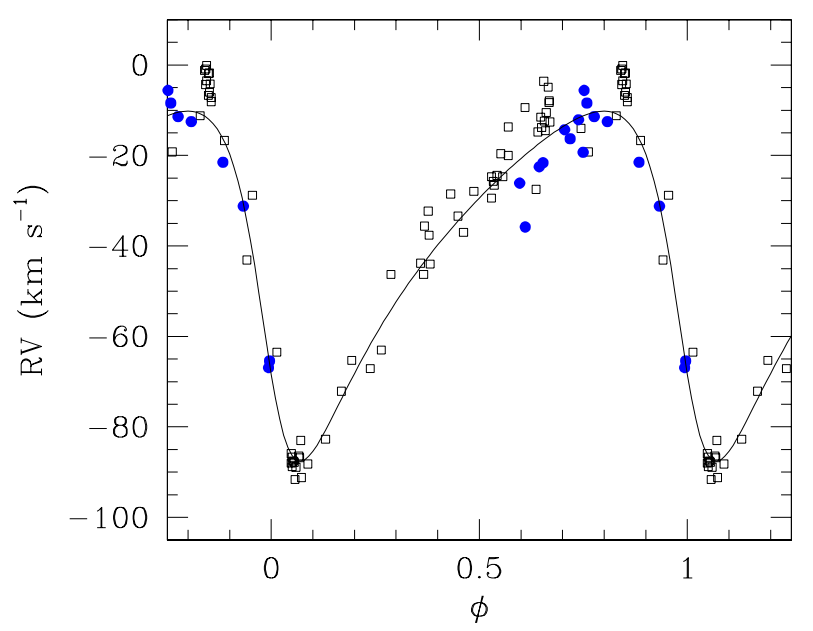

Fig. 1. New SB1 orbital solution of HD 15558, using He II $\lambda 4542$ RVs from De Becker et al. (2006, open squares) and from our new HEROS data (filled circles, see Table B.1). The corresponding orbital elements are given in Table 2.

Willis \& Stickland (1980) noted that the IUE UV spectrum of HD 15570 was intermediate between that of normal Of stars and Wolf-Rayet stars of the WN sequence, thereby suggesting an intermediate evolutionary stage.

Several authors (Hillwig et al. 2006; De Becker et al. 2006, 2009) searched for RV variations that could reveal a spectroscopic binary. Whilst there are some low-level variations in the radial velocities, these variations are not significant and might reflect contamination of the absorption lines by some intrinsically variable wind emission. For instance, based on 68 spectra taken between September 2000 and November 2007, De Becker et al. (2009) reported a RV of $-47.1 \pm 5.8 \mathrm{~km} \mathrm{~s}^{-1}$ for the He II $\lambda 4542$ line.

Polcaro et al. (2003) reported on strong $\mathrm{H} \alpha$ line profile and equivalent width variability of HD 15570 . They claimed variations of the EW of the $\mathrm{H} \alpha$ emission between -3.3 and $-11.7 \AA$, which translate into variations by $40 \%$ of the mass-loss rate (assuming a smooth wind). The He II $\lambda 4686$ emission was found to be considerably less variable (Polcaro et al. 2003). De Becker et al. $(2006,2009)$ confirmed the variability of the emission lines in the spectrum of HD 15570, but at a significantly lower level than indicated by Polcaro et al. (2003).

Even though we only have four HEROS spectra of this star, they are fully consistent with the general description of the spectrum and its variability as given by De Becker et al. (2006) and De Becker et al. (2009). For the RV of the He II $\lambda 4542$ line (see Table B.2), we obtain $-60.3 \pm 3.8 \mathrm{~km} \mathrm{~s}^{-1}$. Whilst this value is more negative than the one given by De Becker et al. (2009), this difference might stem from the slightly asymmetric profile of the line when observed under different spectral resolutions.

Bouret et al. (2012) and Šurlan et al. (2013) performed spectral modelling of the FUV, UV and optical spectrum of HD 15570 using the CMFGEN (Hillier \& Miller 1998) and PoWR (Hamann \& Gräfener 2004) model atmosphere codes, respectively. Whilst Bouret et al. (2012) assumed the wind to host a distribution of optically thin clumps, Šurlan et al. (2013) allowed the clumps to be of arbitrary optical depth. Bouret et al. (2012) had to assume a sub-solar phosphorus abundance to fit the PV $\lambda \lambda$ 1118, 1128 resonance lines, whilst Šurlan et al. (2013) could fit these lines with solar phosphorus abundance. Bouret et al. (2012) inferred $T_{\text {eff }}=38000 \mathrm{~K}, \log L / L_{\odot}=5.94$, and a 

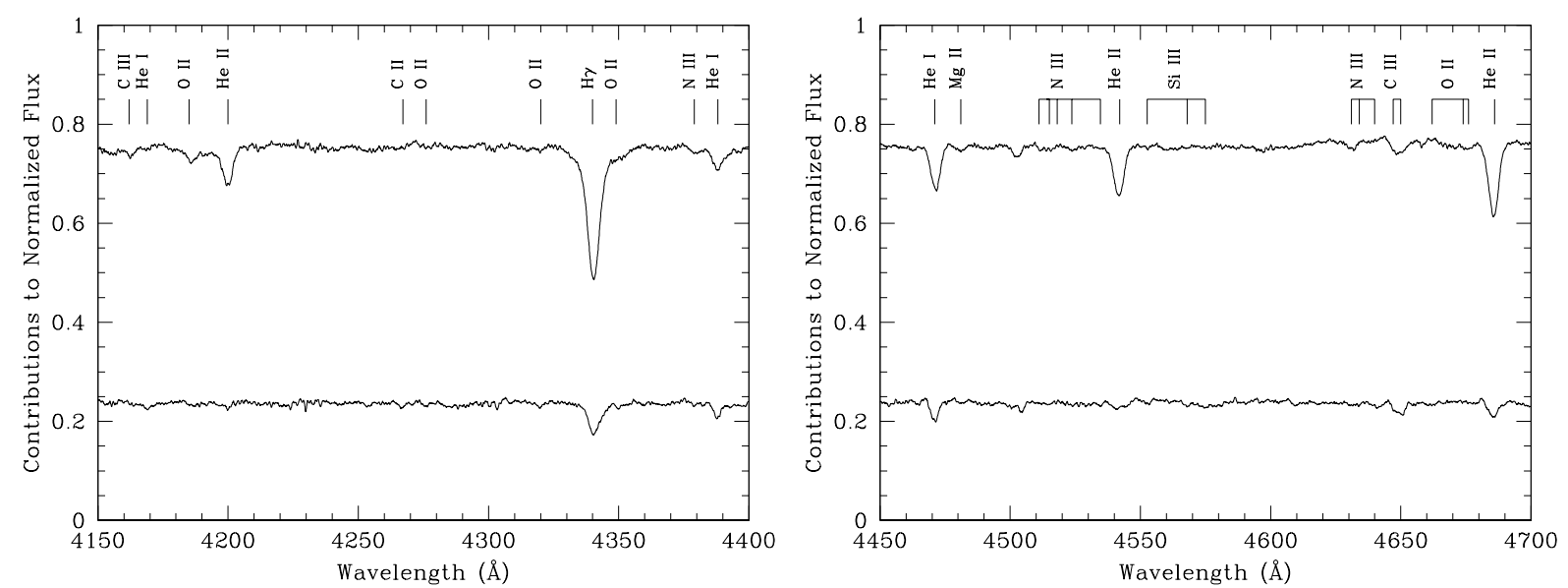

Fig. 2. Blue spectral regions of the components of BD $+60^{\circ} 497$ obtained by disentangling of our HEROS spectra. The primary (top) and secondary (bottom) spectra are shown with their continua normalized to the relative contribution to the combined spectrum.

mass-loss rate $\dot{M}=2.19 \times 10^{-6} M_{\odot} \mathrm{yr}^{-1}$. These authors also noted strongly enhanced $\mathrm{N}$ and strongly depleted $\mathrm{C}$ abundances, which most likely reflect the advanced evolutionary stage of the star. Šurlan et al. (2013) adopted the same effective temperature, luminosity and chemical composition (except for phosphorus) and derived a mass-loss rate of $2.75 \times 10^{-6} M_{\odot} \mathrm{yr}^{-1}$, in good agreement with the value of Bouret et al. (2012).

\section{3. $H D 15629$}

Sota et al. (2011) proposed a spectral-type O4.5 V((fc)) where the "c" tag indicates that the C III $\lambda \lambda 4647-50-51$ emission lines have the same intensity as N III $\lambda$ 4634. Our two HEROS spectra suggest a slightly later spectral type (O5), but otherwise agree with the results of Sota et al. (2011). We find that the Si IV $\lambda 4089$ line is filled up by emission and that the Si IV $\lambda 4116$ line is weakly in emission. Moreover, we note a group of emission lines at 6716,6721 and $6728 \AA$ that we tentatively identify as a blend of C III and N V lines following the discussion for other stars in Walborn (2001).

Repolust et al. (2004) analysed the optical line spectrum of HD 15629 with the FASTWIND (Puls et al. 2005) code and inferred $T_{\text {eff }}=40500 \mathrm{~K}, \log L / L_{\odot}=5.60 \pm 0.13$ and mass-loss rate $\dot{M}=1.28 \times 10^{-6} M_{\odot} \mathrm{yr}^{-1}$ (assuming a smooth wind). A subsequent analysis by Martins et al. (2005) with the CMFGEN code accounting for wind clumping led to similar stellar parameters $\left(T_{\text {eff }}=41000 \mathrm{~K}, \log L / L_{\odot}=5.56\right)$, but a significantly lower mass-loss rate $\left(3.2 \times 10^{-7} M_{\odot} \mathrm{yr}^{-1}\right.$ for a filling factor of 0.1 in the outer regions of the wind).

Neither Hillwig et al. (2006) nor De Becker et al. (2006) found significant and coherent RV variability. Our two HEROS spectra broadly agree with this conclusion (see Table B.3). Therefore, there is currently no evidence for binarity of HD 15629. Peri et al. (2012) studied WISE images that reveal a complex layered structure, making HD 15629 a bow-shock candidate. We stress, however, that the RV of the star does not deviate significantly from that of other O-stars in IC 1805, unlike what would be expected for a runaway star.

\section{4. $B D+60^{\circ} 497$}

This star was found to be a double-lined spectroscopic binary with an orbital period of 3.96 days by Rauw \& De Becker (2004). Based on a set of 16 blue spectra with a resolving power of
8000 obtained with the Aurélie spectrograph at the $1.52 \mathrm{~m}$ telescope at Observatoire de Haute Provence, these authors classified the system as an O6.5 V((f)) + O8.5-9.5 V((f)). They also presented a first orbital solution assuming a circular orbit. Later on, Hillwig et al. (2006) combined the data of Rauw \& De Becker (2004) with their own spectra, which had a resolving power of 5700, to revise the orbital solution. They derived a period of $3.95863 \pm 0.00021$ days and instead favoured an eccentric orbital solution with $e=0.156 \pm 0.019$.

Our new HEROS spectra clearly confirm that this system has an eccentric orbit. We applied our spectral disentangling routine, which is based on the method of González \& Levato (2006), to the HEROS and Aurélie spectra of the system and derived revised radial velocities that are listed in Table B.4. The separated spectra of $\mathrm{BD}+60^{\circ} 497$ (Fig. 2) support the previous spectral classification proposed by Rauw \& De Becker (2004). Based on the Conti (1973) criterion, we infer spectral types O6.5 V((f)) and $08.5 \mathrm{~V}$ for the primary and secondary, respectively.

Using the LOSP code with the newly derived RVs, we then derived a new orbital solution for BD $+60^{\circ} 497$ (see Fig. 3 and Table 3). We considered two different sets of RV data, either restricted to the RVs obtained through spectral disentangling, or also including the RVs quoted by Hillwig et al. (2006). In both cases, we find an eccentricity that matches the value proposed by Hillwig et al. (2006) quite well, whilst the longitude of periastron is significantly larger than the value obtained by Hillwig et al. (2006). This could indicate that the system undergoes a relatively fast apsidal motion. However, by far the largest differences between the two orbital solutions concern the amplitudes of the RV curves. This also leads to somewhat different values of the mass ratio. Additional spectroscopic monitoring at high spectral resolution is probably needed to solve this problem.

\section{5. $B D+60^{\circ} 498$}

This star was classified as O9.7 II-III by Sota et al. (2011). Underhill (1967) reported double lines in the spectrum of $\mathrm{BD}+60^{\circ} 498$, suggesting it to be a binary.

Our 12 HEROS spectra of this star reveal RV changes (measured on H I, He I and He II lines) between -125 and $-11 \mathrm{~km} \mathrm{~s}^{-1}$ with a clear progression from the lowest to the least-negative value over a seven nights interval (see Table B.5 and Fig. 4). This situation suggests that this is a binary system with an orbital period of more than ten days, possibly with a rather eccentric orbit. 

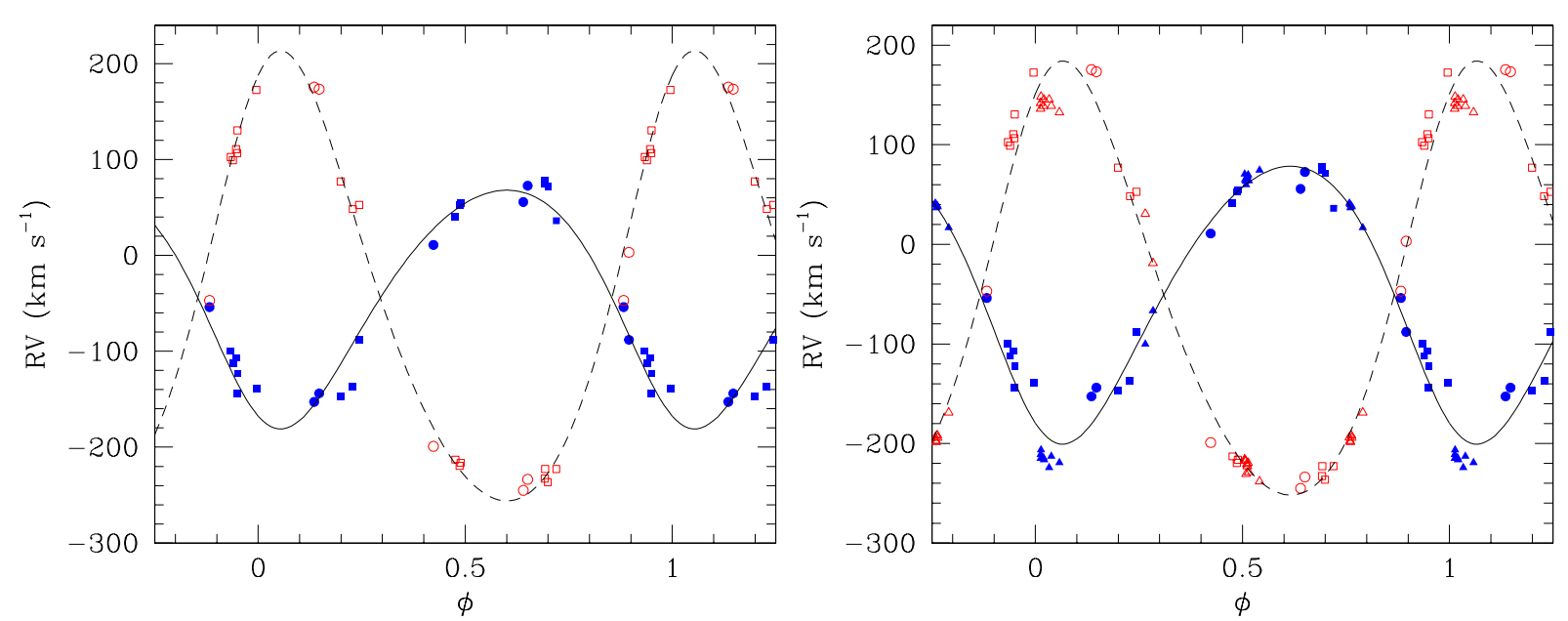

Fig. 3. Left: new orbital solution of $\mathrm{BD}+60^{\circ} 497$, using only RVs obtained by spectral disentangling (see text and Table 3 ). Aurelie data from Rauw \& De Becker (2004) are shown by squares, HEROS data are given by circles. Filled and open symbols stand for the primary and secondary star, respectively. Right: same, but this time also including the RV data from Hillwig et al. (2006, shown by triangles).

Table 3. New orbital solution of BD $+60^{\circ} 497$.

\begin{tabular}{lcc}
\hline \hline & $\begin{array}{c}\text { Aurélie }+ \text { HEROS } \\
\text { data }\end{array}$ & $\begin{array}{c}\text { Aurélie, HEROS } \\
\text { + Hillwig et al. (2006) }\end{array}$ \\
\hline$P_{\text {orb }(\text { days })}$ & $3.959266 \pm 0.000030$ & $3.959259 \pm 0.000024$ \\
$T_{0}(\mathrm{HJD})$ & $2456682.086 \pm 0.071$ & $2456682.037 \pm 0.069$ \\
$e$ & $0.159 \pm 0.039$ & $0.149 \pm 0.017$ \\
$\omega\left(^{\circ}\right)$ & $153.1 \pm 7.2$ & $148.0 \pm 6.9$ \\
$m_{1} / m_{2}$ & $1.88 \pm 0.09$ & $1.56 \pm 0.06$ \\
$K_{1}\left(\mathrm{~km} \mathrm{~s}^{-1}\right)$ & $124.6 \pm 4.9$ & $139.6 \pm 3.5$ \\
$K_{2}\left(\mathrm{~km} \mathrm{~s}^{-1}\right)$ & $234.7 \pm 9.2$ & $218.0 \pm 5.5$ \\
$\gamma_{1}\left(\mathrm{~km} \mathrm{~s}^{-1}\right)$ & $-38.8 \pm 3.6$ & $-43.4 \pm 3.3$ \\
$\gamma_{2}\left(\mathrm{~km} \mathrm{~s}^{-1}\right)$ & $-54.6 \pm 4.6$ & $-61.4 \pm 3.8$ \\
$m_{1} \sin ^{3} i\left(M_{\odot}\right)$ & $11.9 \pm 1.2$ & $11.1 \pm 0.6$ \\
$m_{2} \sin ^{3} i\left(M_{\odot}\right)$ & $6.3 \pm 0.6$ & $7.1 \pm 0.4$ \\
$a \sin i\left(R_{\odot}\right)$ & $27.7 \pm 0.8$ & $27.6 \pm 0.5$ \\
\hline
\end{tabular}

Whilst there are some hints for a secondary spectral signature in He I lines around the most extreme RVs, a far more extensive observing campaign would be needed to derive a full SB2 solution. $\mathrm{BD}+60^{\circ} 498$ thus appears to be the third O-type binary system within IC 1805 . The spectral lines are rather broad, and part of this broadening very likely stems from the blends with the companion's spectral signature. The Conti (1973) criteria applied to the mean spectrum yield a combined spectral type 09.7 in agreement with the result of Sota et al. (2011). However, the strength of the He II $\lambda 4686$ absorption, as well as the ratio between the strengths of Si IV $\lambda 4088$ and He I $\lambda 4143$, suggest a main-sequence luminosity class instead of a giant or bright giant, as proposed by Sota et al. (2011).

\section{6. $B D+60^{\circ} 499$}

Sota et al. (2011) classified this star as O9.5 V, and our HEROS spectra fully agree with this classification. Underhill (1967) suggested that this is a single star.

We have only two HEROS spectra separated by three nights. The spectra reveal very narrow lines. Huang \& Gies (2006) determined a projected rotational velocity of only $18 \mathrm{~km} \mathrm{~s}^{-1}$ for this star, whilst Martins et al. (2015) obtained $30 \mathrm{~km} \mathrm{~s}^{-1}$.

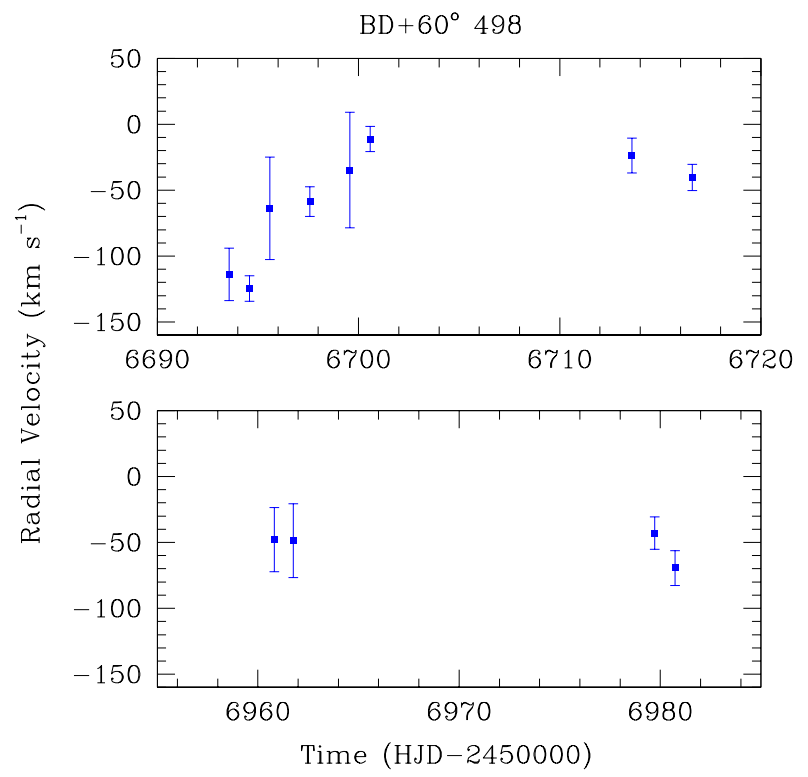

Fig. 4. Radial velocities of $\mathrm{BD}+60^{\circ} 498$ as measured on our HEROS data (see Table B.5). The top panel corresponds to data taken in February 2014, whilst the bottom panel illustrates the data from October and November 2014.

Huang \& Gies (2006) also noted a change in RV (from -66.0 to $-41.3 \mathrm{~km} \mathrm{~s}^{-1}$ ) between their two spectra. Although our data set is clearly not sufficient to rule out binarity, we stress that our data indicate a constant RV of $-46.6 \mathrm{~km} \mathrm{~s}^{-1}$ (Table B.6).

\section{7. $B D+60^{\circ} 501$}

Rauw \& De Becker (2004) provided an O7 V((f)) spectral type, while Sota et al. (2011) classified this star as $\mathrm{O} 7 \mathrm{~V}(n)((\mathrm{f})) z$ where the (n) qualifier stands for lines that are broadened by $v \sin i \sim$ $200 \mathrm{~km} \mathrm{~s}^{-1}$ and the $z$ tag indicates that the He II $\lambda 4686$ line is stronger than both He I $\lambda 4471$ and He II $\lambda 4542$.

Neither Rauw \& DeBecker (2004) nor Hillwig et al. (2006) found significant RV variations for this star, although the mean RVs obtained by these authors significantly differ. 

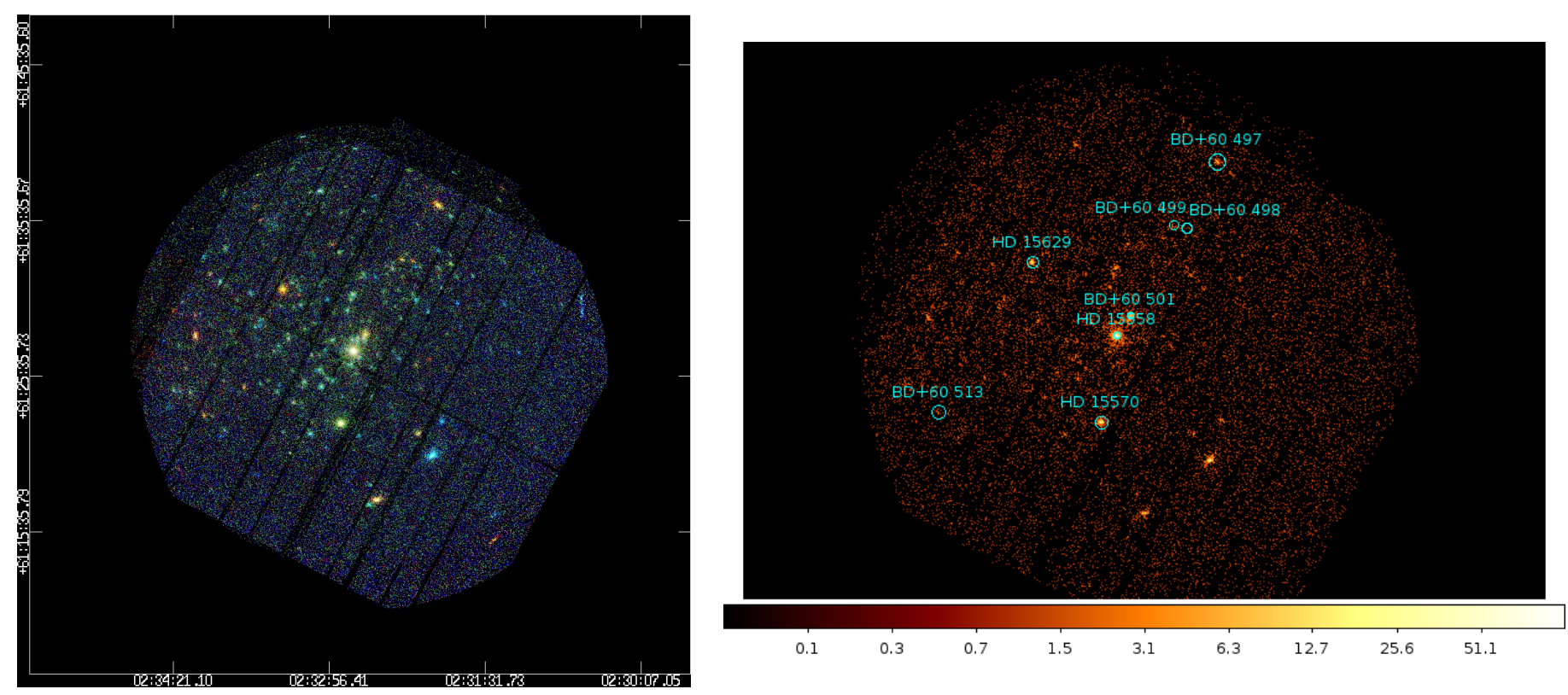

Fig. 5. Left: energy-coded three-colour image of our XMM-Newton observation of IC 1805. Data from the three EPIC cameras were combined and exposure corrected to build this image. Red, green, and blue correspond to photon energies in the ranges [0.5, 1.0], [1.0,2.0], and [2.0, 8.0] keV, respectively. Right: combined EPIC image illustrating the location of the O-type stars in IC 1805.

Rauw \& De Becker (2004) obtained a mean of $-49.9 \pm 2.5$, whilst Hillwig et al. (2006) instead inferred $-57.9 \pm 1.1 \mathrm{~km} \mathrm{~s}^{-1}$.

\section{8. $B D+60^{\circ} 513$}

This star displays broad absorption lines in its optical spectrum indicating that it must be a fast rotator (Rauw \& De Becker 2004; Hillwig et al. 2006). Whilst some RV variations were reported, they most likely result from distortions of the broad lines by intrinsic profile variations (Rauw \& De Becker 2004). The star was classified as O7.5 V((f)) by Rauw \& De Becker (2004) and $07 \mathrm{Vnz}$ by Sota et al. (2011), where the $\mathrm{n}$ qualifier stands for lines that are broadened by $v \sin i \sim 300 \mathrm{~km} \mathrm{~s}^{-1}$. Rauw \& De Becker (2004) obtained a mean RV of $-44.3 \pm 10.5$, whilst Hillwig et al. (2006) instead quoted $-58.6 \pm 2.8 \mathrm{~km} \mathrm{~s}^{-1}$.

In February 2014 we obtained a single HEROS spectrum of the star that confirms the $07.5 \mathrm{Vnz}$ spectral type. There is an Of emission hump between 4600 and $4700 \AA$. Measuring the same spectral lines as Rauw \& De Becker (2004), we obtain a heliocentric RV of $-65.7 \pm 9.7 \mathrm{~km} \mathrm{~s}^{-1}$ where the quoted uncertainty corresponds to the dispersion among the values of the four lines.

\section{X-ray emission in IC 1805}

Figure 5 illustrates the X-ray view of IC 1805 as seen with XMM-Newton. Red, green, and blue correspond to photon energies in the ranges $[0.5,1.0],[1.0,2.0]$, and $[2.0,8.0] \mathrm{keV}$, respectively. The detection algorithm yields a total of 191 detected EPIC sources (see Table A.1). Except for a few soft sources, the brightest X-ray emitters appear yellow and are associated with the O-star members of the cluster. A few bright sources (XID 5, 8,11 ) that are not associated with O-stars appear red in Fig. 5. These are most likely late-type foreground stars (see Sect. 4.3). Some very bright blue sources (XID 3 and 13) could be associated with background AGNs. Finally, many weak sources, most of which display a relatively hard emission (green or even blue in Fig. 5) are scattered throughout the cluster. Most of these sources are very probably pre-main-sequence (PMS) or extragalactic background sources; see below and Sung et al. (in prep.) for a full discussion of their counterparts.

\subsection{O-type stars}

Eight stars of an O-type spectral type are located inside the EPIC field of view (see Fig. 5 and the objects listed in Sect. 3). Two more objects inside the field of view have been classified as $\mathrm{O}$-stars in the literature at some point, but this classification has been revised since then. ALS 7270 (=IC $1805113=$ LS I $+61^{\circ} 277$ ) was listed as O9.5 Ve by Shi \& Hu (1999). However, Negueruela et al. (2004) reclassified this star as B1 Ve rather than $09.5 \mathrm{Ve}$, explaining its non-detection in our XMM-Newton observation. Similarly, BD $+60^{\circ} 512$ is listed in the online table of Reed (2005) as an O6 star. However, the coordinates given by Reed (2005) and the spectral classification refer to the nearby $\mathrm{BD}+60^{\circ} 513$ (see Sect.3). Ishida (1970) instead classified $\mathrm{BD}+60^{\circ} 512$ as a foreground F8 V star. This probably explains why this star is undetected in our XMM-Newton observation.

The fits of the X-ray spectra of the O-stars were made using xspec (Arnaud 1996) version 12.9.0i. The plasma abundances were taken to be solar (Asplund et al. 2009). The interstellar absorption was modelled using the Tübingen-Boulder ISM model (tbabs, Wilms et al. 2000). To evaluate the interstellar neutral hydrogen column density, we adopted the photometric colours of Sung \& Lee (1995) along with the intrinsic colours from Martins \& Plez (2006) and the conversion between colour excess and neutral hydrogen column density of Bohlin et al. (1978). X-ray spectra from massive stars can furthermore be absorbed by the ionized stellar wind. To model such an absorption, we imported the stellar wind absorption model of Nazé et al. (2004) into xspec as a multiplicative tabular model (hereafter labelled wind). Emission from collisionally ionized equilibrium optically thin thermal plasma was modelled using the apec models (Smith $\&$ Brickhouse 2001). We used models computed with ATOMDB v2.0.2. The fits were performed for energy bins between 0.2 and 
HD 15558

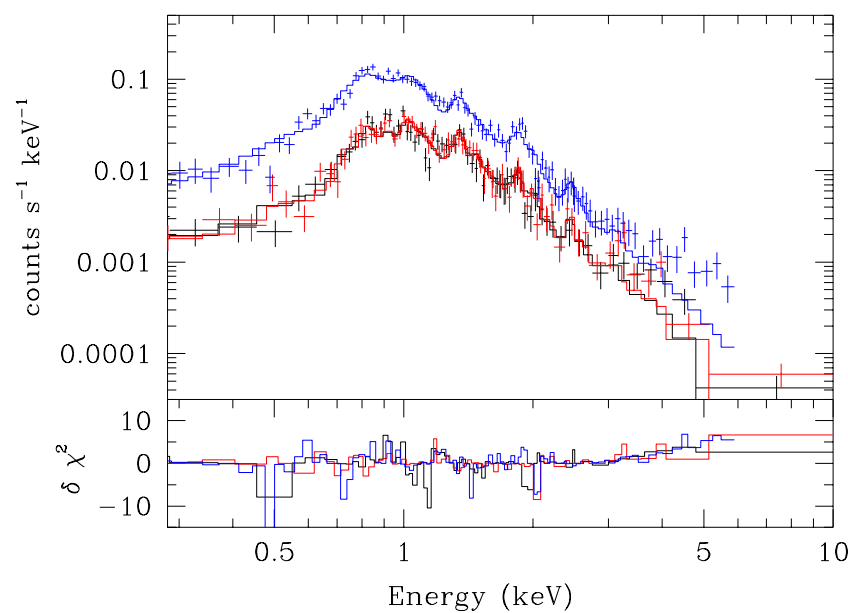

HD 15570

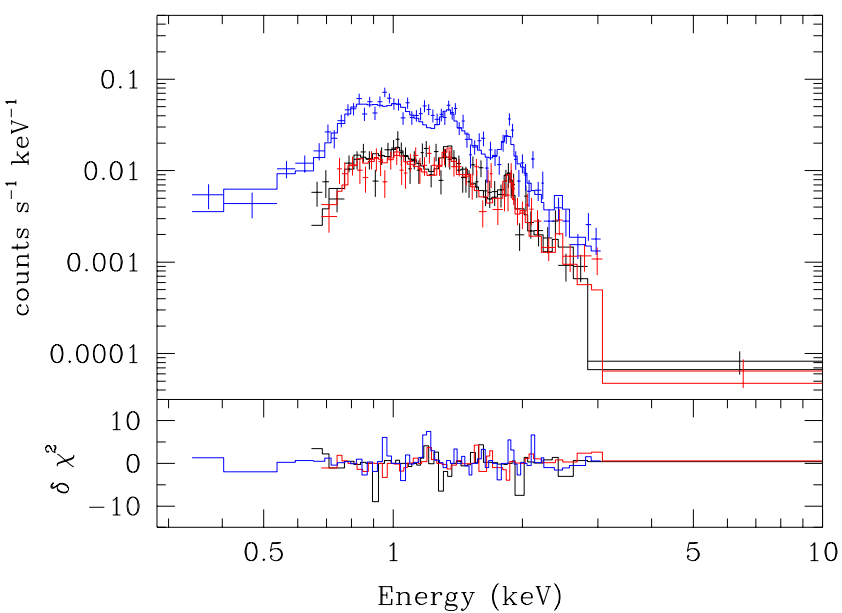

Fig. 6. Left: comparison of the EPIC spectra of HD 15558 with the best-fit model quoted in Table 4. Black, red, and blue colours refer to the spectra obtained with the EPIC-MOS1, MOS2, and pn detectors, respectively. Right: same for HD 15570.

12.0 keV. X-ray fluxes corrected for the interstellar absorption and estimates of their errors were obtained using the cflux command in xspec.

We tested models of the type tbabs $\times$ wind $x$ $\sum_{i=1}^{1 \text { or } 2} \operatorname{apec}\left(\mathrm{T}_{i}\right)$. For low-quality spectra, the data did not allow us to constrain the value of the wind absorption component or the properties of a second plasma component. In those cases, we rather adopted a single-temperature model absorbed by the sole interstellar absorption. The results of our fits are listed in Table 4 and the best fits of the EPIC spectra of HD 15558 and HD 15570 are illustrated in Fig. 6.

Given the sizes of the extraction regions of the XMM-Newton spectra (see Sect. 2.1), we need to consider the possible contamination of the EPIC spectra of the O-type stars by other sources.

HD 15558 is located in a very crowded region of IC 1805: Maíz Apellániz (2010, see also references therein) reported three companions within $10^{\prime \prime} 6$ of the star. The brightest of these objects is about 2.8 mag fainter than HD 15558 in the $z$-band. Ten sources in the Chandra data (Townsley et al. 2014), including the O-star binary itself, lie within the EPIC extraction region (radius of $10^{\prime \prime}$ around HD 15558). These sources are much weaker than HD 15558, however. The net number of counts of the brightest and second-brightest secondary sources is only 2.5 and $2.0 \%$ of that of HD 15558. All other secondary sources are at least a factor of 3 weaker.

Two sources in the ACIS data lie close to the position of $\mathrm{BD}+60^{\circ} 499$. They are located at $1^{\prime \prime} .1$ and $44^{\prime \prime} 6$. The former is most likely the X-ray counterpart of the O-star ${ }^{5}$, whilst the latter ( source $\mathrm{b}=\mathrm{CXO} 023216.25+613312.0$, Townsley et al. 2014) is probably an unrelated PMS star caught during a flare. Its ACIS spectrum presents a much higher plasma temperature than $\mathrm{O}$ stars usually do (see last line of Table 4).

Finally, two secondary sources in the Chandra data, lie within the XMM-Newton spectral extraction region of $\mathrm{BD}+60^{\circ} 501$ (Townsley et al. 2014). The brightest has a net number of ACIS counts that amounts to $18 \%$ of that of $\mathrm{BD}+60^{\circ} 501$ and could thus moderately contribute to the EPIC spectrum of the O-star.

\footnotetext{
5 We note that these objects are located far off-axis in the ACIS data and the PSF is quite heavily elongated, thereby increasing the uncertainties on the source positions.
}
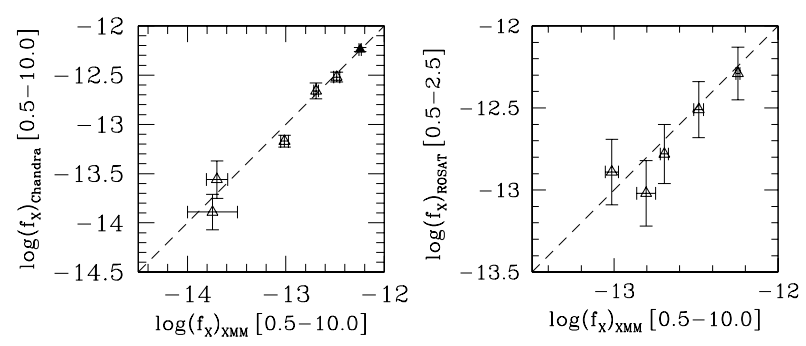

Fig. 7. Left: comparison of the fluxes corrected for ISM absorption of O-type stars in IC 1805 in the $0.5-10 \mathrm{keV}$ energy band as determined with XMM-Newton and Chandra. The filled symbol stands for HD 15558, which is subject to mild pile-up in the Chandra data. The dashed line corresponds to the one-to-one relation. Right: comparison of the $0.5-2.5 \mathrm{keV}$ ROSAT fluxes of O-type stars versus the $0.5-10 \mathrm{keV}$ $X M M-N e w t o n$ fluxes.

We therefore conclude that the EPIC spectra of the O-type stars are only weakly affected by contamination from secondary sources.

\subsubsection{Comparison between different data sets}

Before we correlated the X-ray fluxes with the bolometric fluxes, we compared the absorption-corrected X-ray fluxes inferred from the O-type stars in common between the ROSAT, Chandra and XMM-Newton data. The results are displayed in Fig. 7. The left panel shows excellent agreement between the Chandra and XMM-Newton data. When we discard HD 15558, which is subject to moderate pile-up in the ACIS data, we find an average deviation (in the sense ACIS - EPIC) of $-0.03 \mathrm{dex}^{6}$. This result contrasts with the situation found in Cygnus OB2 (Rauw et al. 2015), where significant differences between the two instruments were found. The right panel of Fig. 7 also indicates a good agreement within errors between the PSPC and EPIC data. The latter comparison also indicates that most of the X-ray emission of the O-type stars in IC 1805 occurs in the soft band.

Another conclusion that can be drawn from Fig. 7 is that there are no large flux changes between the epochs of the various X-ray observations. Furthermore, adopting a significance

6 Including HD 15558 in the analysis does not change the conclusion in any significant way. 


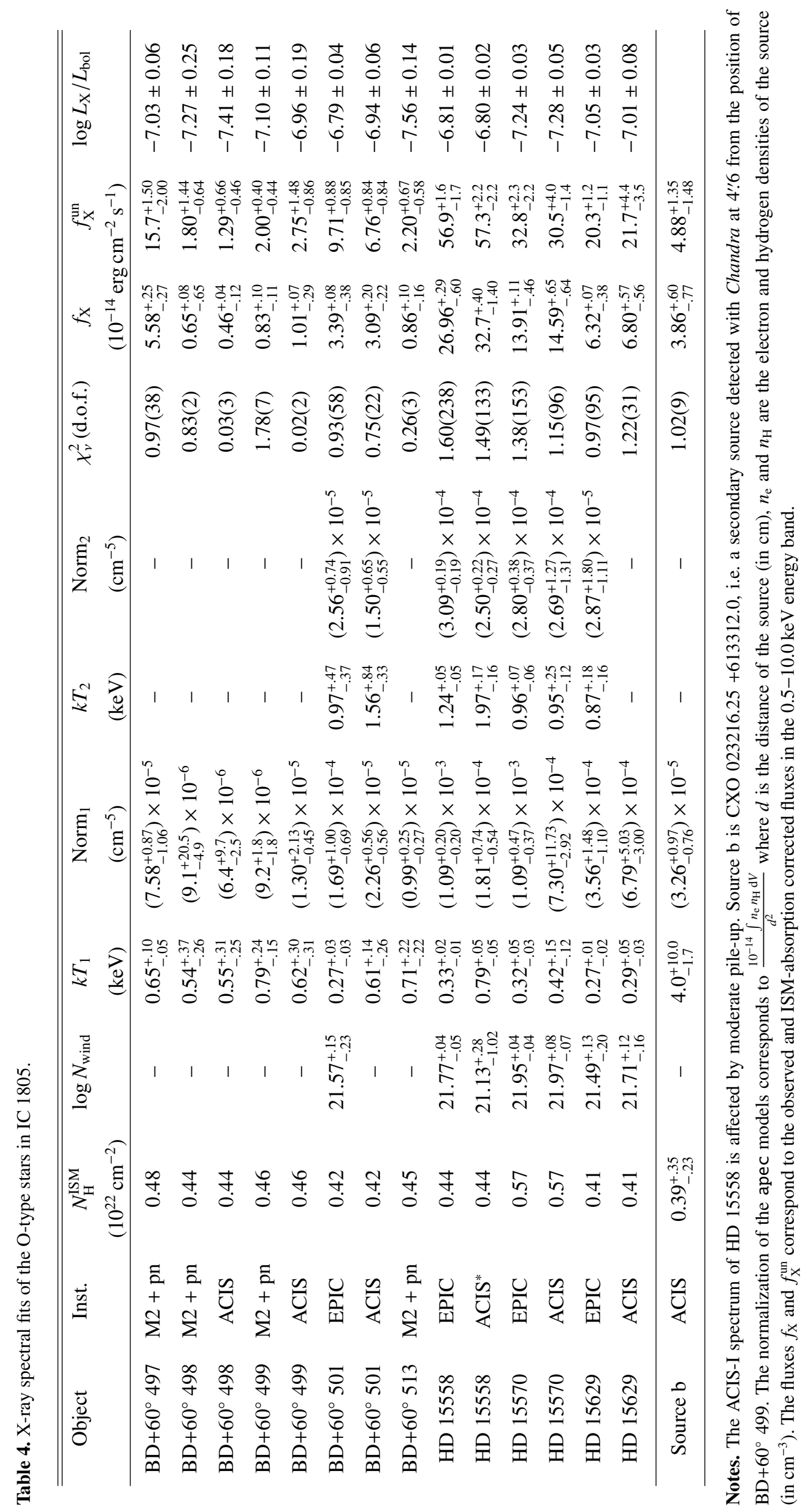



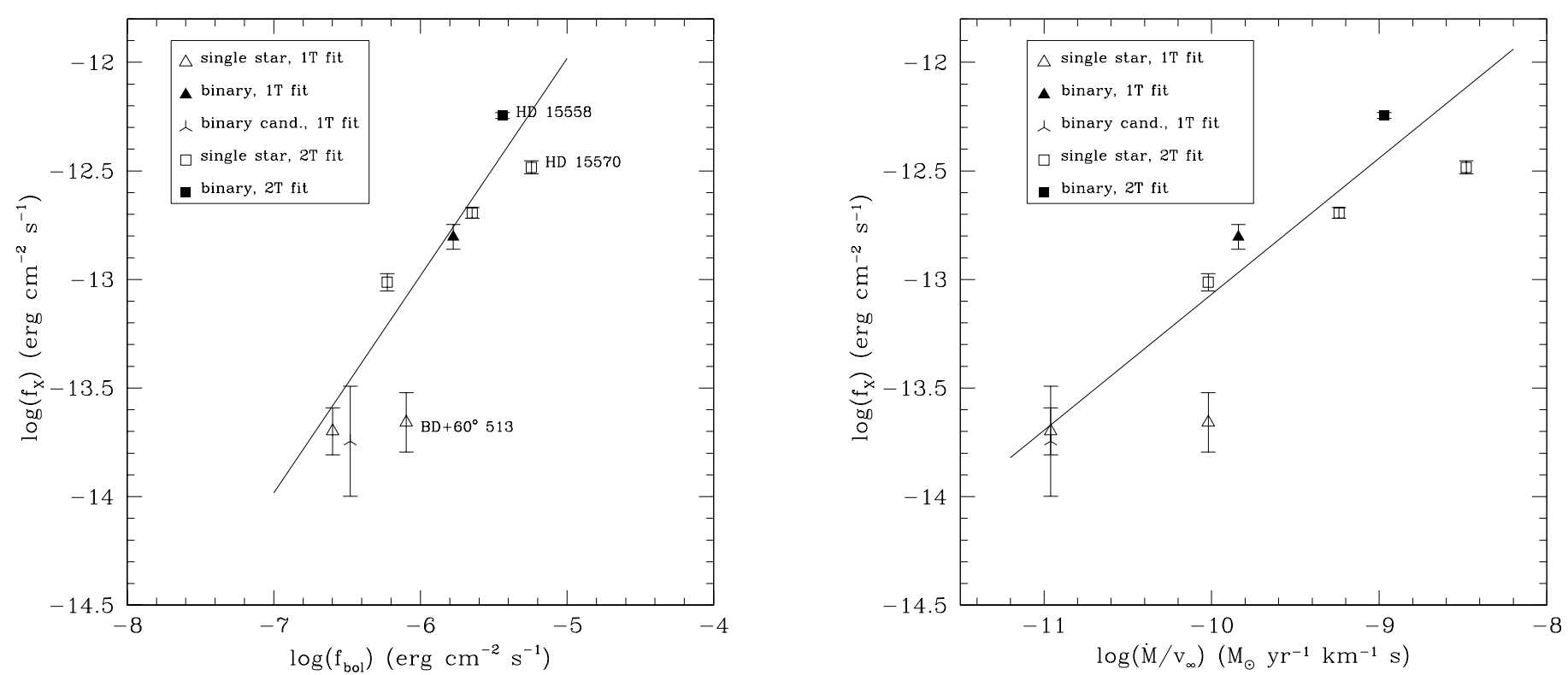

Fig. 8. Left: logarithm of the X-ray fluxes corrected for ISM absorption of O-type stars in IC 1805 as a function of the logarithm of the bolometric fluxes. The solid line yields the best-fit scaling relation (Eq. (1)) between the X-ray and bolometric fluxes considering the full sample of O-type stars, i.e. including binaries as well as single stars. Right: logarithm of the X-ray fluxes corrected for ISM absorption of O-type stars in IC 1805 as a function of $\log \frac{\dot{M}}{v_{\infty}}$. The solid lines yield the theoretical relation $\log f_{\mathrm{X}} \propto(1-m) \log \left(\frac{\dot{M}}{v_{\infty}}\right)$ (Owocki et al. 2013) with $m=0.37$ (Eq. (2)).

level of $1 \%$ in our variability tests (see Sect. 2.1), we found that all O-stars were compatible with a constant emission during the $X M M-N e w t o n$ exposure. Overall, the X-ray emission of the majority of the O-stars in IC 1805 seems constant on short and long timescales.

\subsection{2. $L_{x} / L_{\text {bol }}$ relation of O-type stars}

To build the $L_{\mathrm{X}} / L_{\mathrm{bol}}$ relation of the O-type stars in IC 1805, we first need to establish their bolometric fluxes. For this purpose, we adopted the $B$ and $V$ magnitudes from Sung \& Lee (1995). The extinction $A_{V}$ was obtained assuming $R_{V}=2.9$ (Sung \& Lee 1995) and using the intrinsic colours as well as bolometric corrections as a function of spectral type given by Martins \& Plez (2006). For the two established binary systems, HD 15558 and $\mathrm{BD}+60^{\circ} 497$, we took the contributions from each star into account. De Becker et al. (2006) estimated that the primary of HD 15558 accounts for about 5/6 of the bolometric luminosity of the system. For BD $+60^{\circ} 497$, the studies of Rauw \& De Becker (2004) and Hillwig et al. (2006) agree about an optical brightness ratio of $\sim 0.35$ between the secondary and primary stars, and the bolometric flux was computed accordingly. Finally, for the binary candidate $\mathrm{BD}+60^{\circ} 498$, we currently lack information on the secondary star's spectral type. For this system, we therefore assumed the bolometric correction corresponding to its combined spectral type (see Sect. 3.5).

The X-ray fluxes corrected for ISM absorption found on our $X M M-N e w t o n$ data are plotted as a function of the bolometric fluxes in Fig. 8.

In some massive binary systems, colliding stellar winds provide an excess of X-ray emission in addition to the intrinsic emission generated in the winds of the individual binary components (e.g. Rauw \& Nazé 2016, and references therein). The amount of additional X-ray emission varies tremendously from one object to the other, however, and in recent years it was found that the vast majority of massive binaries are not
X-ray overluminous (e.g. Oskinova 2005; Nazé et al. 2011, 2013a; Rauw et al. 2015). From our current best knowledge of multiplicity among O-stars in IC 1805 (see Sect.3), we expect only three objects $\left(\mathrm{BD}+60^{\circ} 497, \mathrm{BD}+60^{\circ} 498\right.$, and HD 15558) to host potential X-ray emission from colliding winds.

Whilst HD 15558 is indeed the X-ray brightest object in the cluster, neither its X-ray luminosity nor its plasma temperature appear exceptional. In a wide eccentric binary such as HD 15558, the wind interaction region is likely in the adiabatic regime (Stevens et al. 1992). As a result, the X-ray emission from the colliding wind interaction is expected to scale with the inverse of the orbital separation. In the present case, with $e=0.42$, we expect a variation by a factor 2.4 between apastron and periastron. Our XMM-Newton observation $(\phi=0.46)$ and the ROSAT observation $(\phi=0.42)$ were both obtained near apastron when the orbital separation is largest, hence the contribution of the colliding winds should be close to its minimum value. However, the Chandra observation was taken at orbital phase $\phi=0.11$. In principle, we might therefore obtain a rough estimate of the emission level from colliding winds by considering the variations of the X-ray flux between the Chandra and $X M M-N e w t o n$ observation. Unfortunately, the ACIS data are affected by pile-up, which renders such a comparison quite uncertain. At best, we note from our spectral fits that HD 15558 appears indeed brighter during the ACIS observation, but only slightly so (by $21 \%$ in observed flux, and by less than $1 \%$ in ISMcorrected flux), certainly not by a factor 2 . Concerning the other two binaries, we note that none of them appears X-ray overluminous compared to the general trend in Fig. 8. We therefore conclude that to first order, X-ray emission from colliding winds probably does not play a major role in the properties of the X-ray emission of O-stars in IC 1805.

Using the X-ray fluxes corrected for ISM absorption obtained from our XMM-Newton data and the estimates of the bolometric fluxes, we have fitted a simple least-squares scaling 
relation:

$\log L_{\mathrm{X}} / L_{\mathrm{bol}}=-6.98 \pm 0.20$.

Both the average value of $\log L_{\mathrm{X}} / L_{\mathrm{bol}}$ and its dispersion are typical of samples of O-type stars in massive young open clusters (e.g. Sana et al. 2006b; Nazé 2009; Nazé et al. 2011; Rauw et al. 2015). We then investigated the correlation between $f_{\mathrm{X}}$ and $\frac{\dot{M}}{v_{\infty}}$. The quantity $\frac{\dot{M}}{v_{\infty}}$ provides a measure of the mean wind density, and the cooling length of the hot shocked plasma is inversely proportional to this parameter (Hillier et al. 1993). Therefore, it seems quite natural that this quantity should play a role in the $\mathrm{X}$-ray emission. Analysing a sample of 42 ROSAT-PSPC spectra of O-type stars, Kudritzki et al. (1996) empirically derived a relation $L_{\mathrm{X}} \propto\left(\frac{\dot{M}}{v_{\infty}}\right)^{-0.38} L_{\mathrm{bol}}^{1.34}$. Accounting for the dependence of $\frac{\dot{M}}{v_{\infty}}$ on $L_{\text {bol }}$ (see Eq. (32) of Owocki et al. 2013), the Kudritzki et al. (1996) result leads to $L_{\mathrm{X}} \propto\left(\frac{\dot{M}}{v_{\infty}}\right)^{0.67}$. Based on theoretical considerations, Owocki et al. (2013) predicted that the X-ray luminosity scales as $\left(\frac{\dot{M}}{v_{\infty}}\right)^{1-m}$ with $m$ in the range [0.2,0.4]. To check whether such a relation applies to our data, we need to estimate the wind terminal velocities and the mass-loss rates. For the former, we adopted the ratio $v_{\infty} / v_{\mathrm{esc}}=2.6$ along with the escape velocities quoted by Muijres et al. (2012) for the spectral types of the stars in our sample. For the mass-loss rates, we used the values given by Muijres et al. (2012) as a function of spectral type, which correspond to the formalism of Vink et al. (2001) with $v_{\infty} / v_{\mathrm{esc}}=2.6$. For the two double-lined spectroscopic binaries, we considered the spectral type of the primary star, while we used the combined spectral type for the binary candidate $\mathrm{BD}+60^{\circ} 498$ (see Sect. 3). A least-squares fit to the full sample of O-stars in IC 1805 then yields

$\log f_{\mathrm{X}}=(0.63 \pm 0.21) \log \left(\frac{\dot{M}}{v_{\infty}}\right)_{\text {theor }}-6.81 \pm 2.10$.

We have to stress that the values of the mass-loss rates and terminal velocities that we use here are theoretical values for a given spectral type that do not account for the effect of clumping. When we consider the two stars in our sample (HD 15570 and HD 15629) for which dedicated model atmosphere fits accounting for clumping are available in the literature (Bouret et al. 2012; Martins et al. 2015), we find that these clumping-corrected mass-loss rates are lower by a factor 2.9 to 4.6. When we assume, however, that the clumping correction to be applied to the Vink et al. (2001) mass-loss rates is essentially independent of spectral type, the overall trend in the right panel of Fig. 8 should be correct. Relation (2) nicely agrees with the empirical relation found by Kudritzki et al. (1996). Our best-fit $m$ value $(0.37 \pm 0.21)$ is well inside the range predicted by Owocki et al. (2013). It is lower than what we found for the Cyg OB2 stars $(0.52 \pm 0.10$, Rauw et al. 2015), although both values overlap within their errors.

\subsubsection{A break in the $L_{X} / L_{\text {bol }}$ relation for $O$ If $^{+}$stars?}

The two data points that deviate most from relation(2) are $\mathrm{BD}+60^{\circ} 513$ at the faint end, and to a lesser extent, HD 15570 at the brighter end. If we discard these two stars, the best-fit relation becomes

$\log f_{\mathrm{X}}=(0.71 \pm 0.14) \log \left(\frac{\dot{M}}{v_{\infty}}\right)_{\text {theor }}-5.92 \pm 1.40$.
The coefficients in Eqs. (2) and (3) overlap within the errors. $\mathrm{BD}+60^{\circ} 513$, which has the highest projected rotational velocity among the O-type stars in IC 1805 , is also quite faint in its $L_{\mathrm{X}} / L_{\text {bol }}$ ratio. On the other hand, HD 15570, which has $\log L_{\mathrm{X}} / L_{\text {bol }}=-7.24 \pm 0.03$, is indeed the $\mathrm{O} 4 \mathrm{If}^{+}$star we are interested in. Does this result imply that $\mathrm{O} \mathrm{If}^{+}$stars, and early-O If supergiants in general, are indeed X-ray faint? To answer this question, we first considered other studies of luminous O-supergiants in Galactic young open clusters.

Nazé et al. (2011) studied the $L_{\mathrm{X}} / L_{\text {bol }}$ relation for massive stars in the Carina complex. If we focus on the earliest supergiants in their sample, we find $\log L_{\mathrm{X}} / L_{\mathrm{bol}}=-6.97$ for HD 93129A (O2 If* + O3.5 V) and -7.21 for $\mathrm{Cl}^{*}$ Trumpler 14 MJ 257 (O3/4 If). Generally speaking, presumably single O-stars with spectral types $\mathrm{O} 2$ - O5.5 in the Carina Nebula were found to display $\log L_{\mathrm{X}} / L_{\mathrm{bol}}=-7.25 \pm 0.22$. Hence, there was no evidence for a significant underluminosity of the earliest $\mathrm{O}$-supergiants in this cluster.

Nazé et al. (2013a) studied the X-ray emission of the massive star population of the open cluster HM 1, which harbours two early-O If ${ }^{+}$stars, VB 4 and VB 5, which are of spectral types $\mathrm{O} 4 \mathrm{If}^{+}$and $\mathrm{O} 5 \mathrm{If}^{+}$, respectively. Both were found to have rather low $\log L_{\mathrm{X}} / L_{\mathrm{bol}}$ values of -7.4 and -7.3 for VB 4 and VB 5, respectively. However, HM 1 is subject to very heavy interstellar absorption, and Nazé et al. (2013a) noted that, given the limited signal-to-noise ratio of their EPIC spectra, this large absorption could lead to an overestimate of the plasma temperature and accordingly to an underestimate of the X-ray flux for these and other stars of the same cluster. Therefore, although this result indicates a relative $\mathrm{X}$-ray underluminosity of $\mathrm{O} \mathrm{If}^{+}$, it is not sufficient to conclude that this effect is indeed real.

Finally, Rauw et al. (2015) reported $\log L_{\mathrm{X}} / L_{\mathrm{bol}}=-7.03$ for the O3 If* star Cyg OB2 \#7, whilst the full sample of O-type stars in Cyg OB2 (excluding the very bright, overluminous colliding wind systems Cyg OB2 \#5, 8a and 9) yields $\log L_{\mathrm{X}} / L_{\mathrm{bol}}=$ $-7.18 \pm 0.21$. Hence, the $\mathrm{O}$ supergiant is fully consistent with the overall relation.

Returning to the specific case of IC 1805, we examined the possible causes for the deviations seen in Fig. 8. For this purpose, we considered the recent work of Gayley (2016), who proposed a first attempt to estimate the X-ray emergence efficiency from the winds of all types of non-magnetic single massive stars. The emergence efficiency $\eta_{\mathrm{X}}$ is defined as the ratio between the emerging X-ray luminosity and the total excess turbulent energy flux injected into the wind. Based on first principles, Gayley (2016) provided a simple expression of $\eta_{\mathrm{X}}$ (his Eq. (11)) as a function of the ratio between the velocity jump needed to account for the mean X-ray photon energy and the wind terminal velocity, on the one hand, and the wind optical depth $\tau(L)$ at some specific distance $L$ in the wind ${ }^{7}$, on the other hand. The latter parameter is a proxy for the mass-loss rate. We solved Eq. (11) of Gayley (2016) assuming $m=0.37$ (see Eq. (2)) to estimate $\eta_{\mathrm{X}}$ as a function of $\tau(L)$. We used these results to compare the emergence efficiency for $1 \mathrm{keV}$ photons in HD 15629 $\left(\log L_{\mathrm{X}} / L_{\mathrm{bol}}=-7.05\right)$ and HD $15570\left(\log L_{\mathrm{X}} / L_{\mathrm{bol}}=-7.24\right)$. Both stars are most likely single (see Sect. 3), and their stellar and wind parameters have been determined with the same model atmosphere code (Martins et al. 2005; Bouret et al. 2012). Assuming $L=1 R_{*}$ (Gayley 2016), we can then estimate values of $\tau(L) \sim 0.34$ for HD 15629 and $\sim 1.66$ for HD 15570 .

7 This distance scale parameter is the correlation length between the fast wind and the slower clumps. It also corresponds to the porosity length of the wind (Gayley 2016). 
These values translate into X-ray emergence efficiencies of $35 \%$ and $16 \%$, respectively. Hence, we would expect a 2.2 times (0.34 dex) lower efficiency for the denser wind of HD 15570. Similar conclusions are obtained when we consider the mean photon energies of the apec models in Table 4 instead of a photon energy of $1 \mathrm{keV}$. Hence, the mild X-ray underluminosity of HD 15570 can very likely be traced to its specific stellar and wind parameters.

\subsection{B- and A-type stars}

Of the 191 sources detected in the EPIC field of view, 13 have an optical counterpart of spectral type B, three are classified as A-type stars, and two as Herbig Ae/Be star. Spectroscopic surveys of A- and B-type stars in IC 1805 are probably far from complete. Shi \& Hu (1999), who provide one of the most extensive spectroscopic surveys of this cluster, list 78 B-type stars in IC 1805, 49 of which lie inside our EPIC field of view. Of these 49 B-stars, 37 have a spectral type earlier than or equal to B4, whilst the remaining 12 have later spectral types. Of the $13 \mathrm{X}$-ray sources that have a B-type optical counterpart, 10 have been classified by Shi \& Hu (1999), 9 of them are early B-type stars, whilst only 1 has a spectral type later than B4. We thus have detection rates of $\sim 24 \%$ for B0-B4 stars and $\sim 8 \%$ for B5-B9.5 stars. This situation is very much reminiscent of that reported by Sana et al. (2006b) for the NGC 6231 cluster: a detection rate of $\sim 24 \%$ for B0-B4 stars (15 objects out of 63) and a significant drop (to $\sim 7 \%, 2$ objects out of 28 ) at later subtypes. A higher detection efficiency of 50\% (54 objects out of 108) for B-type stars up to spectral type B5 (included) was achieved in the Chandra legacy survey of Cyg OB2 (Rauw et al. 2015). This could be due to the higher sensitivity of Chandra for faint pointlike sources.

Three of the EPIC sources with probable A- or B-type optical counterparts were sufficiently bright to produce at least 400 EPIC counts, and we therefore extracted and analysed their spectra (see Sect. 2.1). The spectra were fitted within xspec with either absorbed optically thin thermal plasma apec models or absorbed power-law spectra. The results of these fits are quoted in Table 5 along with the spectral fits of other bright sources in the field of view that are discussed in Sect. 4.3.

XMMU J023240.7+612801 (XID 16) most probably corresponds to IC 1805 143, an early B-star with literature spectral classifications of B1 V (Massey et al. 1995), B0.5 V (Shi \& Hu 1999), or B0.3 V (Huang \& Gies 2006). The best EPIC position is at 1 ".4 from CXOU 023240.76+612800.2. Although this separation is relatively large for a source located within $1^{\prime}$ of the Chandra aimpoint, their association is highly likely. A second Chandra source (CXOU 023240.79+612758.5) lies in the $X M M-N e w t o n$ extraction region, but it is twice as distant and more than 50 times fainter. The absorption and X-ray flux of the target were larger during the Chandra observations: in the power-law fits, absorption doubled and flux quadrupled, while the slope did not change significantly ${ }^{8}$.

The optical counterpart of XMMU J023243.3+612803 (XID 18) is classified as a late B-type star in Sung et al. (in prep.) and as an A6 star by Wolff et al. (2011). The EPIC spectra of this object are well fitted by a moderate absorption, close to the typical value for the IC 1805 cluster, and a rather high temperature.

\footnotetext{
8 The usual degeneracy of the fits between a highly absorbed warm plasma and a less absorbed hot plasma prohibits clear conclusions from the thermal fits regarding changes of the absorption.
}

The Chandra source CXOU 023243.21+612804.5 lies at 1'!3 distance, which is a non-negligible separation especially since the Chandra source was not too far off-axis $\left(<1^{\prime}\right)$. Nevertheless, if both X-ray sources are associated, then the X-ray flux appears to have quadrupled in the XMM-Newton observation.

XMMU J023243.7+612631 (XID 19) corresponds to IC 1805152 classified as B3 V by Shi \& Hu (1999). Three Chandra sources are located within the extraction region of XID 19: CXOU 023243.54+612632.0 (at 1".6), CXOU 023243.39+612635.0 (at 4".2), and CXOU 023243.54+ 612636.8 (at 5'!3). While the separation is non-negligible, the $X M M-N e w t o n$ source probably corresponds to the former source, which is 30 times brighter than each of the latter two objects. The $\mathrm{X}$-ray properties (flux, absorption slightly larger than that of the cluster, moderate $\Gamma$ ) appear to have remained relatively constant, within the errors, between the two observations.

A- and B-type stars are usually not expected to be strong intrinsic X-ray emitters. Except for the earliest spectral types, they lack the strong winds that produce the shocks responsible for the X-ray emission of O-type stars. At the same time, except for the latest spectral types, these stars also lack the convective outer layer where the interplay between convection and rotation leads to a dynamo mechanism able to sustain an X-ray bright corona. Observations indeed confirm that A- and B-type stars are generally X-ray dark: based on data from the ROSAT AllSky Survey, Schröder \& Schmitt (2007) found that stars of spectral type B1-A9 have low, but non-zero, X-ray detection rates, roughly between 10 and $15 \%$. The origin of the X-ray emission of the detected sources is not clear: it could either be intrinsic to the $\mathrm{AB}$ stars or arise from unseen late-type companions. A similar situation holds for Herbig Ae/Be stars, where the actual origin of the X-ray emission (intrinsic to the star, produced in a magnetic star/disk interaction, or due to an unresolved latetype pre-main-sequence companion) remains a puzzle (Stelzer et al. 2009). However, the high incidence of binary companions (Duchêne 2015) in Herbig Ae/Be stars suggests that their X-ray emission might be associated, at least to some extent, with unseen low-mass companions.

One of the most promising scenarios for an intrinsic emission of $\mathrm{AB}$ stars is the magnetically confined wind shock (MCWS) model (Babel \& Montmerle 1997; ud-Doula \& Owocki 2002). Robrade (2016) discussed the X-ray emission of magnetic $\mathrm{Ap} / \mathrm{Bp}$ stars. This author notably found that X-ray detections are more frequent among the earliest and most luminous stars in his sample, although there is a large scatter in X-ray luminosity for a given bolometric luminosity. This situation favours an intrinsic origin of the X-ray emission of Ap/Bp stars, possibly related to the MCWS scenario as the more luminous stars have stronger winds that could more easily lead to a significant X-ray emission when interacting with the magnetic field. However, a strong (kilo-Gauss) magnetic field does not provide a sufficient condition for an intrinsic X-ray emission. In a sample of ten magnetic late-B and early-A stars, Czesla \& Schmitt (2007) failed to detect X-rays for four stars. Another argument against magnetic fields as the main explanation for the X-ray emission of B-type stars comes from the incidence rate of magnetic fields among OB stars. Sensitive spectropolarimetric surveys have established this rate to be about 6-8\% (Wade et al. 2014; Fossati et al. 2015), which is well below the X-ray detection rate. Hence, whilst magnetically confined winds could indeed explain a few percent of the X-ray detections of early B-type stars, they certainly cannot account for the full set of detections.

The three sources for which we were able to extract EPIC spectra display quite hard spectra, consistent with the 
G. Rauw and Y. Nazé: The X-ray view of IC 1805

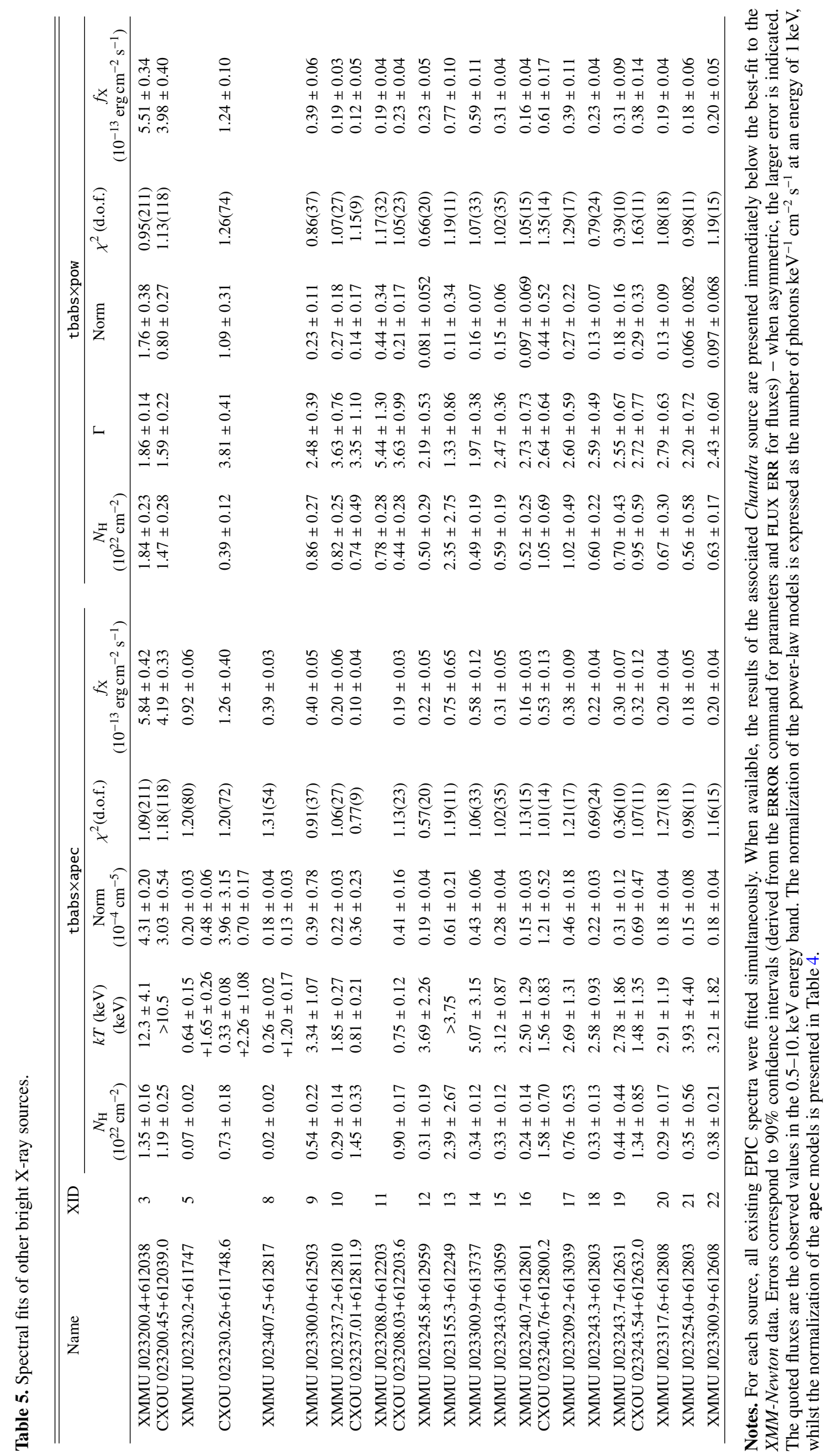

A82, page 13 of 19 

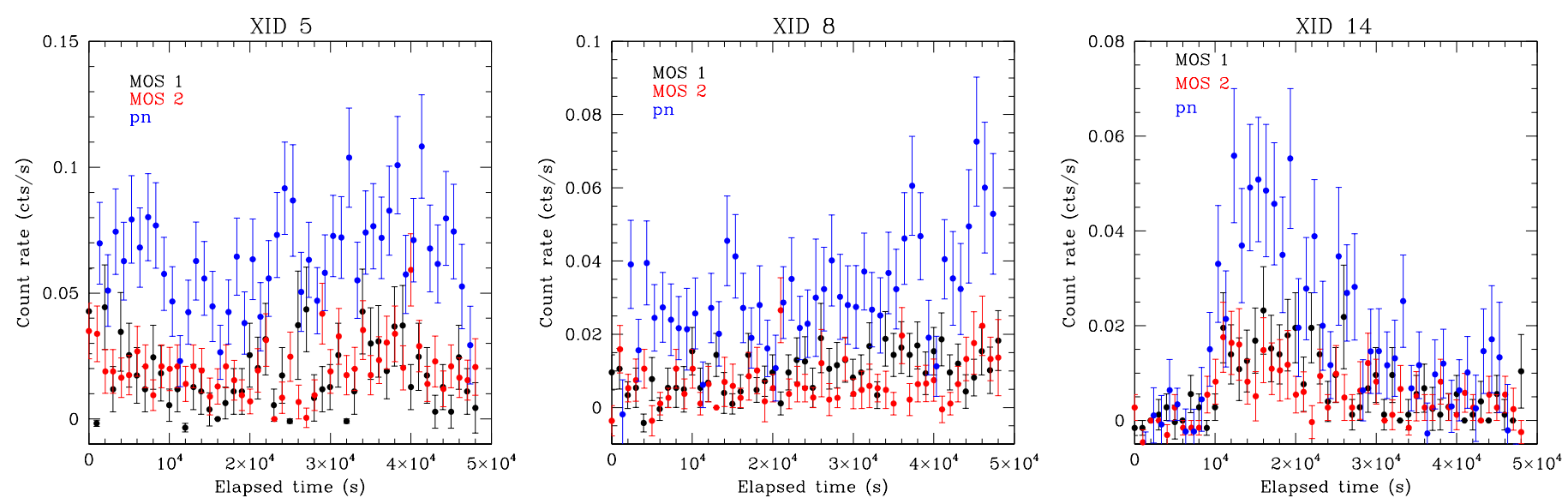

Fig. 9. EPIC light curves of three secondary sources in the field of IC 1805: XID 5, 8, and 14, from left to right. Black, red, and blue correspond to count rates measured with the EPIC-MOS1, MOS2, and pn cameras, respectively. The light curves were extracted with time bins of 1 ks. Time zero corresponds to the beginning of our XMM-Newton observation.

generally high plasma temperatures found for low-mass premain-sequence (PMS) stars (see Sect. 4.3). Moreover, the hardening and flux increase of XID 16 between the Chandra and $X M M-N e w t o n$ observations may indicate a flaring event. These characteristics thus favour an interpretation of the X-ray emission arising from a late-type PMS companion and not from the $\mathrm{AB}$ star itself.

\subsection{Other bright $X$-ray sources in the field of view}

As explained in Sect.2.1, we extracted EPIC spectra for 17 sources that are not O-type stars but were bright enough (they had at least 400 EPIC counts) to perform a (rough) spectral analysis. A cross-correlation with the Chandra source list of Townsley et al. (2014) was made for these sources, with an association considered secure whenever the separation was smaller than $1^{\prime \prime}$. For those targets for which Townsley et al. (2014) reported at least 100 ACIS net counts (in the $0.5-8.0 \mathrm{keV}$ energy band), we also extracted the ACIS spectra (see Sect.2.2). The spectral fitting was made within xspec considering optically thin thermal plasma apec models and the possibility of nonthermal X-ray emission (represented by a power-law spectrum). Table 5 lists the results of both types of fits, and we now briefly examine each source in turn, except for the three sources that have been discussed in Sect. 4.2.

The third brightest X-ray source in the field of view (XID 3) is XMMU J023200.4+612038, which corresponds to CXOU 023200.45+612039.0 (Townsley et al. 2014), probably a young stellar object (YSO) according to Broos et al. (2013). Sung et al. (in prep.) classify this object as a class I YSO. However, since most YSOs in IC 1805 are rather of class II, the authors caution that it is more likely for this source to be of a different nature, for example extragalactic. This possibility is indeed supported by our X-ray analysis. The best-fit absorbing column exceeds that of the cluster, and the fit by a thermal emission component yields a rather high temperature $(>8 \mathrm{keV})$, while the power-law model has a $\Gamma \sim 2$ in the XMM-Newton data, two properties in line with an extragalactic nature. While the source appeared constant, at the $1 \%$ significance level, during the XMM-Newton exposure, the overall flux increased by nearly $40 \%$ in the EPIC data compared to the Chandra-ACIS data.

The spectrum of XMMU J023230.2+611747 (XID 5) is clearly better fitted by thermal emission models, and two components are needed to achieve a good fit. This source corresponds to CXOU 023230.26+611748.6 (Townsley et al. 2014) and IC 1805 132, an object considered as a star by Broos et al. (2013) and Sung et al. (in prep.). The latter authors suggest this is a foreground F/G-type star ${ }^{9}$. The fitted temperatures are moderate, and the absorption column density is quite low, typical of nearby stellar sources. The usual trade-off between a more absorbed warm solution and a less absorbed hot solution is found, with Chandra and $X M M$-Newton data each converging to one of these cases, respectively (see Table 5). The analysis of the XMM-Newton light curve (especially that of EPIC-pn) rejects the constancy hypothesis at the $1 \%$ significance level, but the behaviour of the light curve appears more complex than a simple trend or a single large flare (see Fig. 9). In addition, the overall flux appeared to have decreased by about $40 \%$ in the XMM-Newton exposure compared to the Chandra observation, although the error bar is large, hence variation is at the edge of significance.

The spectrum of XMMU J023407.5+612817 (XID 8) is quite similar to that of the previous source, the best fit indicating two moderate temperatures with little absorption, compatible with the foreground late-type star nature pointed out by Sung et al. (in prep.). The emission constancy is rejected at the $1 \%$ significance level for the EPIC-pn light curve, and the EPIC-pn and EPIC-MOS1 light curves are both significantly better fitted by increasing trends than by constants (see Fig. 9). Unfortunately, this target is located outside the Chandra field of view.

The flux of XMMU J023300.0+612503 (XID 9) appears to significantly increase during the XMM-Newton observation. Its X-ray emission appears somewhat absorbed, with either a rather steep slope for a power-law model or a very high temperature for a thermal plasma model. This source corresponds to CXOU 023259.92+612503.1 in Townsley et al. (2014), whose optical/IR counterpart is classified as a young star in Broos et al. (2013). Sung et al. (in prep.) also find that this counterpart lies within the PMS locus of IC 1805. With only 26 net Chandra counts (Townsley et al. 2014), a meaningful spectral comparison cannot be performed for this object, but we note that Townsley et al. (2014) attributed a photon flux of $1.62 \times 10^{-6} \mathrm{ph} \mathrm{cm}^{-2} \mathrm{~s}^{-1}$ with a median energy of $1.73 \mathrm{keV}$ to this source, corresponding to a flux ${ }^{10}$ of $\sim 4.5 \times 10^{-15} \mathrm{erg} \mathrm{cm}^{-2} \mathrm{~s}^{-1}$. The target would thus

\footnotetext{
9 According to Vasilevskis et al. (1965) and Sanders (1972), the cluster membership probability of IC 1805132 is indeed $0 \%$.

10 We note that this procedure yielded a flux close to those found in spectral fits for the six Chandra sources for which meaningful spectral
} information could be extracted. 
have brightened by an order of magnitude between the two observations. In this context, it may be noted that constancy was also rejected at the $0.5 \%$ significance level in the Chandra data (Townsley et al. 2014).

Three Chandra sources are found within the XMM-Newton extraction region of XMMU J023237.2+612810 (XID 10): CXOU 023237.01+612811.9, CXOU 023236.57+612813.4, and CXOU 023238.26+612807.6 (ordered by increasing separation). The former object clearly dominates the emission of the region in the Chandra data, as it has nearly 120 net counts, while the two other sources display fewer than seven counts together. In addition, these two faint sources are at least twice as distant from the XMM-Newton position. While its position is shifted by 2 ".5 compared to that of the XMM-Newton target, a quite high value for the Chandra source within $1^{\prime}$ of the aimpoint, the spectral properties of the best-fit power laws are similar in both Chandra and XMM-Newton exposures, suggesting that the two objects are associated. The source flux may have doubled in the $X M M$-Newton observation, but this variation is again at the edge of significance in view of the large error bars. The number of counts were here too low for a meaningful light-curve analysis, but we note that a Kolmogorov-Smirnov test on the Chandra data rejected the constancy null hypothesis at the $0.5 \%$ significance level (Townsley et al. 2014). Sung et al. (in prep.) find its optical/IR counterpart to lie within the PMS locus of the cluster.

The spectrum of XMMU J023208.0+612203 (XID 11) appears much better fitted by a power law than by a thermal plasma emission model. This source corresponds to CXOU 023208.03+612203.6, which is considered to be a star (Broos et al. 2013; Sung et al., in prep., it appears in the PMS locus in this latter reference, but the possibility of a foreground late-type star cannot be excluded). Straižys et al. (2013) assigned a $\mathrm{K} 4 \mathrm{~V}$ photometric spectral type to the optical counterpart. The source flux is similar in the Chandra and XMM-Newton observations, but we note that a Kolmogorov-Smirnov test on the Chandra data rejected the constancy null hypothesis at the $<0.01 \%$ significance level (Townsley et al. 2014).

XMMU J023245.8+612959 (XID 12) has an absorption similar to that of the cluster, and it appears moderately hard $(k T \sim 4 \mathrm{keV}, \Gamma \sim 2)$. Three Chandra sources are found within the XMM-Newton extraction region of this source: CXOU 023245.77+612959.4, CXOU 023245.84+ 612955.3, and CXOU 023246.78+613001.2 (in order of increasing separation). The former object clearly dominates the emission of the region in the Chandra data, as it has more than 60 counts, while the two other sources display $\sim 7$ counts together (Townsley et al. 2014). Moreover, these two faint objects are at 3".6 and 7" distance from the XMM-Newton position, respectively. The photon flux and median energy reported by Townsley et al. (2014) suggest a doubling of the X-ray flux in the XMM-Newton observation. Sung et al. (in prep.) find the optical/IR counterpart to lie within the PMS locus, while Broos et al. (2013) suggested a (young) stellar nature for the X-ray source.

XMMU J023155.3+612249 (XID 13) displays a highly absorbed and hard X-ray emission. The temperature associated with a thermal model fitting is unrealistically high. This is not surprising because this target corresponds to CXOU 023155.35+612249.8, which is classified as an AGN in Broos et al. (2013), a scenario reinforced by the lack of optical counterparts in the sensitive survey of Sung et al. (in prep.). The photon flux and median energy reported by Townsley et al. (2014) suggest a tripling of the X-ray flux in the XMM-Newton observation.
The light curve of XMMU J023300.9+613737 (XID 14) clearly displays a moderate flare during the observation (Fig. 9). Spectral fitting yields an absorption close to that of the cluster, while the intrinsic emission is moderately hard. Only one Chandra source lies within the XMM-Newton extraction region, the young star CXOU 023300.58+613737.1, but it is offset by $2 \prime \prime 8$, which is large even for an object at $10^{\prime}$ off-axis angle in the ACIS data. If the two sources correspond to the same object, then the X-ray flux would have changed by a factor of 20. Broos et al. (2013) suggested a stellar nature for the X-ray source, and Sung et al. (in prep.) report an optical counterpart that lies in the PMS locus of the cluster.

XMMU J023243.0+613059 (XID 15) has a spectrum similar to that of the previous source. In Simbad, it appears close to 2MASS J02324314+6130587 (which has a likely YSO classification in Broos et al. 2013, and a class II YSO classification in Sung et al., in prep.). Straižys et al. (2013) reported a WISE counterpart, suggesting this object to be an Ae/Be highmass YSO (see also Sect. 4.2). Its extraction region encompasses four Chandra sources: CXOU 023243.14+613058.6 (at 0'.75), CXOU 023243.01+613102.5 (at 3".2), CXOU 023243.36+ 613056.3 (at 3".5), and CXOU 023242.08+613105.3 (at 9'.5). The former and latter sources have a similar number of counts, but the latter one is quite far away from the XMM-Newton position; the two other sources are fainter by a factor of $\sim 5$. If the $X M M$-Newton source corresponds to the closest Chandra source, then the photon flux and median energy reported by Townsley et al. (2014) suggest a fourfold increase of its flux.

XMMU J023209.2+613039 (XID 17) displays a quite hard spectrum, with an absorption stronger than the typical cluster value and a high temperature/photon index. Its extraction region encompasses two Chandra sources: CXOU 023209.23+613040.8 (at 1'!1 - not too high considering the ACIS off-axis angle of $5^{\prime}$ ) and CXOU 023207.89+613047.4, which is five times fainter in Townsley et al. (2014) and at a very large angular distance of $12^{\prime \prime} .5$ from the XMM-Newton position. The former Chandra source is a likely YSO (Broos et al. 2013), more precisely classified as a class II YSO by Sung et al. (in prep.); it is also known as 2MASS J02320924+6130404 and was found to be a WISE source probably associated with a YSO (Straižys et al. 2013). Compared to Chandra observations (see photon flux and median energy in Townsley et al. 2014), its $\mathrm{X}$-ray flux has quadrupled.

XMMU J023317.6+612808 (XID 20) displays an absorption compatible with that of the cluster and moderately high temperature and $\Gamma$. In its extraction region lie two Chandra sources CXOU 023317.49+612808.4 and CXOU 023316.53+612809.5, the latter being 20 times fainter and 7 times farther away from the XMM-Newton position. Despite a separation of $1^{\prime \prime} .2$, it is therefore reasonable to consider the former Chandra source as the counterpart of the XMM-Newton source. The photon flux and median energy reported by Townsley et al. (2014) suggest that the object was somewhat brighter during the XMM-Newton observation. The optical/IR counterpart is a likely YSO (Broos et al. 2013) lying in the PMS locus (Sung et al., in prep.). Straižys et al. (2013) quoted a photometric spectral type $G$ for this object.

With a similar spectrum as the previous object, XMMU J023254.0+612803 (XID 21) is most probably associated with CXOU 023253.96+612804.3, which lies at $1^{\prime \prime}$ (a second source in the extraction region, CXOU 023254.24+ 612814.6 is 30 times fainter in Chandra exposure and 10 times farther away). Its optical/IR counterpart is a young star (Broos et al. 2013) lying in the PMS locus (Sung et al., in prep.), and 
its XMM-Newton flux is somewhat higher than the one derived from the photon flux of Townsley et al. (2014).

Again displaying a similar spectrum as the previous sources, XMMU J023300.9+612608 (XID 22) is the probable counterpart of CXOU 023300.79+612609.6 (which was at nearly $3^{\prime}$ off-axis in the Chandra data, explaining the larger separation - 1'!3 with the XMM-Newton position). A second source, CXOU 023300.57+612606.8, appears both eight times fainter and twice more distant, rendering an association less likely. The optical/IR counterpart is a likely YSO (Broos et al. 2013) lying in the PMS locus (Sung et al., in prep.) and the X-ray flux is about eight times higher in the XMM-Newton observation than in the Chandra data.

In summary, XID 3 and 13 are probably extragalactic background sources, whilst XID 5 and 8 are probably associated with foreground late-type stars. The remaining bright X-ray sources are most probably PMS members of IC 1805. Megeath et al. (2008) quoted a typical distance for the W4 region of $2 \mathrm{kpc}$. Adopting this distance and taking an average neutral hydrogen column density of $0.4 \times 10^{22} \mathrm{~cm}^{-2}$ along with an average plasma temperature of $k T=2.7 \mathrm{keV}$ (see Table 5), we can convert the observed fluxes into intrinsic fluxes and thereby estimate X-ray luminosities. For the $\mathrm{X}$-ray brightest PMS candidates, we found $f_{\mathrm{X}} \simeq 0.5 \times 10^{-13} \mathrm{erg} \mathrm{cm}^{-2} \mathrm{~s}^{-1}$ (Table 5) which then translates into $L_{\mathrm{X}} \simeq 3.5 \times 10^{31} \mathrm{erg} \mathrm{s}^{-1}$. Whilst this is a relatively high value, it is not unusual for PMS stars in young open clusters, especially during flares (Sana et al. 2007; Rauw 2011). We note also that our cross-correlation with catalogues from the literature (see Table A.1) resulted in the association of six X-ray sources with low-mass YSOs identified with WISE (Straižys et al. 2013). Additional support for the conclusion that the bulk of the sources are associated with PMS stars comes from the correlation with deep, sensitive photometry (see Sung et al., in prep.).

\section{Summary and conclusions}

We have presented new X-ray observations and optical spectroscopy of the very young open cluster IC 1805. We improved the orbital solutions of the two previously known O-star binary systems, HD 15558 and $\mathrm{BD}+60^{\circ} 497$, and found evidence that $\mathrm{BD}+60^{\circ} 498$ is a third O-star binary. With this information, we then studied the X-ray emission of the O-star population of IC 1805. X-ray emission from colliding winds in known binary systems was found to play only a marginal role in determining the X-ray luminosity. The X-ray luminosities of O-type stars in IC 1805 follow the well-known canonical scaling relation with bolometric luminosity $\log L_{\mathrm{X}} / L_{\text {bol }}=-6.98 \pm 0.20$. We paid particular attention to HD 15570, an $\mathrm{O} 4 \mathrm{If}^{+}$star, to search for evidence that the dense stellar wind reduces the level of emerging X-ray emission. For this star we obtained $\log L_{\mathrm{X}} / L_{\mathrm{bol}}=-7.24 \pm 0.03$, which is below the scaling relation of the O-stars in the cluster, although at only 1.3 times the dispersion of the latter relation. Such a deviation is compatible with theoretical expectations based on the stellar and wind properties of the star. Considering results for other very early and luminous $\mathrm{O}$-supergiants in the literature, however, we found no clear observational evidence that the most luminous $\mathrm{O}$-supergiants as a class are systematically X-ray underluminous. The reason for this is the relatively small amplitude of the expected reduction effect when compared to the dispersion around the scaling relation.

In addition to the O-type stars, our XMM-Newton X-ray data revealed many weaker sources in the field of view of IC 1805. We discussed the X-ray spectra and light curves of the brightest of these objects. Except for a few field interlopers, most of the sources are probably low-mass pre-main-sequence stars belonging to IC 1805. Finally, whilst we detected about $25 \%$ of the spectroscopically classified B-type stars in the cluster, the properties of the detected sources are in most cases compatible with $\mathrm{X}$-ray emission from otherwise undetected low-mass PMS companions, thus suggesting that the X-ray detections of B- and Atype stars in IC 1805 are due to such low-mass companions.

Acknowledgements. We are grateful to Prof. Hwankyung Sung for sharing some of his results with us before publication. Our thanks go to the referee of our paper for a swift and helpful report. We acknowledge support through an ARC grant for Concerted Research Actions, financed by the French Community of Belgium (Wallonia-Brussels Federation), from the Fonds de la Recherche Scientifique (FRS/FNRS), as well as through an XMM PRODEX contract (Belspo). The TIGRE facility is funded and operated by the universities of Hamburg, Guanajuato and Liège. This research has made use of the SIMBAD database, operated at CDS, Strasbourg, France.

\section{References}

Antipin, S. V. 2008, Peremennye Zvezdy Prilozhenie 8, 19A

Arnaud, K. A. 1996, in Astronomical Data Analysis Software and Systems V, eds. G. Jacoby, \& J. Barnes, ASP Conf. Ser., 101, 17

Asplund, M., Grevesse, N., Sauval, A. J., \& Scott, P. 2009, ARA\&A, 47, 481

Babel, J., \& Montmerle, T. 1997, ApJ, 485, L29

Baumgardt, H., Dettbarn, C., \& Wielen, R. 2000, A\&AS, 146, 251

Berghöfer, T. W., Schmitt, J. H. M. M., Danner, R., \& Cassinelli, J. P. 1997, A\&A, 322, 167

Bieging, J. H., Abbott, D. C., \& Churchwell, E. B. 1989, ApJ, 340, 518

Bohlin, R. C., Savage, B. D., \& Drake, J. F. 1978, ApJ, 224, 132

Bouret, J.-C., Hillier, D. J., Lanz, T., \& Fullerton, A. W. 2012, A\&A, 544, A67

Broos, P. S., Getman, K. V., Povich, M. S., et al. 2013, ApJS, 209, 32

Conti, P. S. 1973, ApJ, 179, 181

Conti, P. S., Hanson, M. M., Morris, P. W., Willis, A. J., \& Fossey, S. J. 1995, ApJ, 445, L35

Czesla, S., \& Schmitt, J. H. M. M. 2007, A\&A, 465, 493

De Becker, M. 2013, New Astron., 25, 7

De Becker, M., Rauw, G., Manfroid, J., \& Eenens, P. 2006, A\&A, 456, 1121

De Becker, M., Rauw, G., \& Linder, N. 2009, ApJ, 704, 964

Duchêne, G. 2015, Ap\&SS, 355, 291

Feigelson, E. D., Townsley, L. K., Broos, P. S., et al. 2013, ApJS, 209, 26

Feldmeier, A., Puls, J., \& Pauldrach, A. W. A. 1997, A\&A, 322, 878

Fossati, L., Castro, N., Schöller, M., et al. 2015, A\&A, 582, A45

Garmany, C. D., \& Massey, P. 1981, PASP, 93, 500

Gayley, K. G. 2016, Adv. Space Res., 58, 719

González, J. F., \& Levato, H. 2006, A\&A, 448, 283

Gosset, E., Royer, P., Rauw, G., Manfroid, J., \& Vreux, J.-M. 2001, MNRAS, 327,435

Gosset, E., Nazé, Y., Claeskens, J.-F., et al. 2005, A\&A, 492, 685

Hamann, W.-R., \& Gräfener, G. 2004, A\&A, 427, 697

Harnden, F., R., Jr., Branduardi, G., Gorenstein, P., et al. 1979, ApJ, 234, L51

Heck, A., Manfroid, J., \& Mersch, G. 1985, A\&AS, 59, 63

Hillier, D. J., \& Miller, D. L. 1998, ApJ, 496, 407

Hillier, D. J., Kudritzki, R. P., Pauldrach, A. W., et al. 1993, A\&A, 276, 117

Hillwig, T. C., Gies, D. R., Bagnuolo, W. G., et al. 2006, ApJ, 639, 1069

Hinkle, K., Wallace, L., Valenti, J., \& Harmer, D. 2000, Visible and Near Infrared Atlas of the Arcturus Spectrum 3727-9300 ̊, eds. K. Hinkle, L. Wallace, J.

Valenti, \& D. Harmer (San Francisco: ASP)

Huang, W., \& Gies, D. R. 2006, ApJ, 648, 580

Ignace, R., Oskinova, L. M., \& Foullon, C. 2000, MNRAS, 318, 214

Ishida, K. 1970, PASP, 22, 277

Jansen, F., Lumb, D., Altieri, B., et al. 2001, A\&A, 365, L1

Kudritzki, R. P., Palsa, R., Feldmeier, A., Puls, J., \& Pauldrach, A. W. 1996, in

Röntgenstrahlung from the Universe, eds. H. U. Zimmermann, J. Trümper, \& H. Yorke, MPE Rep., 263, 9

Lindgren, B. W. 1976, Statistical Theory - 3rd edn. (New York: Mc Millan Pub.)

Maíz Apellániz, J. 2010, A\&A, 518, A1

Martins, F., \& Plez, B. 2006, A\&A, 457, 637

Martins, F., Schaerer, D., Hillier, D. J., et al. 2005, A\&A, 441, 735

Martins, F., Hervé, A., Bouret, J.-C., et al. 2015, A\&A, 575, A34

Massey, P., Johnson, K. E., \& Degioia-Eastwood, K. 1995, ApJ, 454, 151

Megeath, S. T., Townsley, L. K., Oey, M. S., \& Tieftrunk, A. R. 2008, in

Handbook of Star Forming Regions Vol. I, ed. B. Reipurth, ASP, 264

Mernier, F., \& Rauw, G. 2013, New Astron., 20, 42 
Mittag, M., Hempelmann, A., González-Pérez, J. N., \& Schmitt, J. H. M. M 2010, Adv. Astron., 101502

Muijres, L. E., Vink, J. S., de Koter, A., Müller, P. E., \& Langer, N. 2012, A\&A, 537, A37

Nazé, Y. 2009, A\&A, 506, 1055

Nazé, Y., Rauw, G., Vreux, J.-M., \& De Becker, M. 2004, A\&A, 417, 667

Nazé, Y., Broos, P. S., Oskinova, L., et al. 2011, ApJS, 194, 7

Nazé, Y., Rauw, G., Sana, H., \& Corcoran, M. F. 2013a, A\&A, 555, A83

Nazé, Y., Oskinova, L. M., \& Gosset, E. 2013b, ApJ, 763, 143

Nazé, Y., Petit, V., Rinbrand, M., et al. 2014, ApJS, 215, 10

Negueruela, I., Steele, I. A., \& Bernabeu, G. 2004, Astron. Nachr., 325, 749

Oskinova, L. M. 2005, MNRAS, 361, 679

Oskinova, L. M. 2015, in Wolf-Rayet Stars, eds. W.-R. Hamann, A. Sander, \& H. Todt, 295

Oskinova, L. M., Ignace, R., Hamann, W.-R., Pollock, A. M. T., \& Brown, J. C. 2003, A\&A, 402, 755

Owocki, S. P., Sundqvist, J. O., Cohen, D. H., \& Gayley, K. G. 2013, MNRAS, 429, 3379

Peri, C. S., Benaglia, P., Brookes, D. P., Stevens, I. R., \& Isequilla, N. L. 2012, A\&A, 538, A108

Pittard, J. M., \& Parkin, E. R. 2010, MNRAS, 403, 1657

Polcaro, V. F., Viotti, R., Norci, L., et al. 2003, in International Conference on magnetic fields in O, B and A stars, eds. L. A. Balona, H. F. Henrichs, \& T. Medupe, ASP Conf. Series, 305, 377

Puls, J., Urbaneja, M. A., Venero, R., et al. 2005, A\&A, 435, 669

Rauw, G. 2011, A\&A, 536, A31

Rauw, G., \& De Becker, M. 2004, A\&A, 421, 693

Rauw, G., \& Nazé, Y. 2016, Adv. Space Res., 58, 761

Rauw, G., Nazé, Y., Wright, N. J., et al. 2015, ApJS, 221, 1

Reed, B. C. 2005, AJ, 130, 1652

Repolust, T., Puls, J., \& Herrero, A. 2004, A\&A, 415, 349

Robrade, J. 2016, Adv. Space Res., 58, 727

Sana, H., Gosset, E., \& Rauw, G. 2006a, MNRAS, 371, 67

Sana, H., Rauw, G., Nazé, Y., Gosset, E., \& Vreux, J.-M. 2006b, MNRAS, 372, 661

Sana, H., Rauw, G., Sung, H., Gosset, E., \& Vreux, J.-M. 2007, MNRAS, 377, 945
Sanders, W. L. 1972, A\&A, 16, 58

Schmitt, J. H. M. M., Schröder, K.-P., Rauw, G., et al. 2014, Astron. Nachr. 335, 787

Schröder, C., \& Schmitt, J. H. M. M. 2007, A\&A, 475, 677

Sciortino, S., Vaiana, G. S., Harnden, F. R. Jr., et al. 1990, ApJ, 361, 621

Shi, H. M., \& Hu, J. Y. 1999, A\&AS, 136, 313

Skrutskie, M. F., Cutri, R. M., Stiening, R., et al. 2006, AJ, 131, 1163

Smith, R. K., \& Brickhouse, N. S. 2001, ApJ, 556, L91

Sota, A., Maíz Apellániz, J., Walborn, N. R., et al. 2011, ApJS, 193, 24

Stelzer, B., Robrade, J., Schmitt, J. H. M. M., \& Bouvier, J. 2009, A\&A, 493, 1109

Stevens, I. R., Blondin, J. M., \& Pollock, A. M. T. 1992, ApJ, 386, 265

Straižys, V., Boyle, R. P., Jnusz, R., Laugalys, V., \& Kazlauskas, A. 2013, A\&A, 554, A3

Strüder, L., Briel, U., Dennerl, K., et al. 2001, A\&A, 365, L18

Sung, H., \& Lee, S.-W. 1995, J. Korean Astron. Soc., 28, 119

Šurlan, B., Hamann, W.-R., Aret, A., et al. 2013, A\&A, 559, A130

Townsley, L. K., Broos, P. S., Garmire, G. P., et al. 2014, ApJS, 213, 1

Turner, M. J. L., Abbey, A., Arnaud, M., et al. 2001, A\&A, 365, L27

ud-Doula, A., \& Nazé, Y. 2016, Adv. Space Res., 58, 680

ud-Doula, A., \& Owocki, S. P. 2002, ApJ, 576, 413

Underhill, A. B. 1967, in Determination of Radial Velocities and their Applications, IAU Symp., 30, 167

Vasilevskis, S., Sanders, W. L., \& Van Altena, W. F. 1965, AJ, 70, 806

Vink, J. S., de Koter, A., \& Lamers, H. J. G. L. M. 2001, A\&A, 369, 574

Wade, G. A., Grunhut, J., Alecian, E., et al. 2014, in Magnetic Fields throughout Stellar Evolution, IAU Symp., 302, 265

Walborn, N. R. 2001, in Eta Carinae and Other Mysterious Stars: The Hidden Opportunities of Emission Spectroscopy, eds. T. R. Gull, S. Johannson, \& K. Davidson, ASP Conf. Ser., 242, 217

Wessolowski, U. 1996, in Röntgenstrahlung from the Universe, eds. H. U. Zimmermann, J. Trümper, \& H. Yorke, MPE Rep., 263, 75

Willis, A. J., \& Stickland, D. J. 1980, MNRAS, 190, 27

Wilms, J., Allen, A., \& McCray, R. 2000, ApJ, 542, 914

Wolfe, R. H., Jr., Horak, H. G., \& Storer, N. W. 1965, in Modern Astrophysics A Memorial to Otto Struve, ed. M. Hack (New York: Gordon \& Breach), 251 Wolff, S. C., Strom, S. E., \& Rebull, L. M. 2011, ApJ, 726, 19 


\section{Appendix A: List of X-ray sources detected with XMM-Newton}

Table A. 1 provides the full catalogue of X-ray sources detected with the EPIC instruments onboard XMM-Newton ordered by increasing right ascension. The coordinates of the sources were cross-correlated with the optical and IR catalogues of Straižys et al. (2013), Wolff et al. (2011), and the SIMBAD catalogue. We adopted in each case a correlation radius of 4 ", which is generally well adapted for EPIC data. For a more detailed comparison, we refer to the work of Sung et al. (in prep.), who present the results of a cross-correlation with their own photometric catalogue, which is much deeper than the catalogues used here. Adopting the same 4 " radius for cross-correlation with the 2MASS (Skrutzkie et al. 2006) catalogue results in $72 \%$ of the $\mathrm{X}$-ray sources with a single IR counterpart and an additional 5\% with two IR counterparts.

\section{Appendix B: Newly determined radial velocities}

In this section we provide the journal of the new optical spectra of the O-type stars obtained with the HEROS spectrograph at the $1.2 \mathrm{~m}$ TIGRE telescope along with the RVs inferred from these data.

Table B.1. RVs of the He II $\lambda 4542$ line as measured on the HEROS spectra of HD 15558. Typical uncertainties are $5 \mathrm{~km} \mathrm{~s}^{-1}$.

\begin{tabular}{cc}
\hline \hline HJD-2 450 000 & $\begin{array}{c}\mathrm{RV}_{1} \\
\mathrm{~km} \mathrm{~s}^{-1}\end{array}$ \\
\hline 6512.867 & -26.1 \\
6518.819 & -35.8 \\
6533.823 & -22.5 \\
6537.853 & -21.6 \\
6560.899 & -14.3 \\
6566.885 & -16.3 \\
6575.830 & -12.1 \\
6580.770 & -19.3 \\
6581.901 & -5.6 \\
6584.809 & -8.4 \\
6592.667 & -11.4 \\
6606.784 & -12.5 \\
6640.688 & -21.5 \\
6662.591 & -31.2 \\
6689.592 & -66.9 \\
6690.645 & -65.4 \\
\hline
\end{tabular}

Table B.2. Newly determined RVs of HD 15570.

\begin{tabular}{cccc}
\hline \hline HJD-2 450000 & \multicolumn{3}{c}{ RV $\left(\mathrm{km} \mathrm{s}^{-1}\right)$} \\
& He II $\lambda 4542$ & N III $\lambda 4634$ & N III $\lambda 4641$ \\
\hline 6509.918 & -61.0 & -69.0 & -73.3 \\
6519.877 & -65.2 & -58.9 & -74.5 \\
6680.564 & -56.6 & -59.3 & -72.2 \\
6958.815 & -58.3 & -61.6 & -70.5 \\
\hline Mean & $-60.3 \pm 3.8$ & $-62.2 \pm 4.7$ & $-72.6 \pm 1.7$ \\
\hline
\end{tabular}

Table B.3. Newly determined RVs of HD 15629.

\begin{tabular}{cccc}
\hline \hline HJD-2450000 & \multicolumn{3}{c}{ RV $\left(\mathrm{km} \mathrm{s}^{-1}\right)$} \\
& He II $\lambda 4542$ & N III $\lambda 4634$ & N III $\lambda 4641$ \\
\hline 6680.658 & -53.8 & -58.5 & -79.1 \\
6958.757 & -52.7 & -78.7 & -78.2 \\
\hline Mean & $-53.3 \pm 0.8$ & $-68.6 \pm 14.3$ & $-78.7 \pm 0.6$ \\
\hline
\end{tabular}

Table B.4. Newly determined RVs of BD $+60^{\circ} 497$.

\begin{tabular}{cccc}
\hline \hline HJD-2450000 & $\begin{array}{c}\mathrm{RV}_{1} \\
\mathrm{~km} \mathrm{~s}^{-1}\end{array}$ & $\begin{array}{c}\mathrm{RV}_{2} \\
\mathrm{~km} \mathrm{~s}^{-1}\end{array}$ & Instrument \\
\hline 2520.644 & -107.1 & 110.7 & $\mathrm{~A}$ \\
2523.600 & 74.4 & -222.8 & $\mathrm{~A}$ \\
2524.552 & -99.3 & 102.7 & $\mathrm{~A}$ \\
2527.555 & 78.3 & -232.7 & $\mathrm{~A}$ \\
2528.533 & -112.1 & 99.2 & $\mathrm{~A}$ \\
2529.562 & -147.3 & 77.0 & $\mathrm{~A}$ \\
2531.543 & 71.5 & -236.4 & $\mathrm{~A}$ \\
2532.534 & -122.6 & 106.6 & $\mathrm{~A}$ \\
2533.638 & -137.3 & 48.4 & $\mathrm{~A}$ \\
2916.583 & -143.5 & 130.4 & $\mathrm{~A}$ \\
2918.667 & 40.9 & -212.9 & $\mathrm{~A}$ \\
2919.632 & 36.2 & -222.7 & $\mathrm{~A}$ \\
2922.677 & 54.4 & -216.3 & $\mathrm{~A}$ \\
2925.665 & -87.9 & 52.7 & $\mathrm{~A}$ \\
2928.641 & -139.1 & 172.5 & $\mathrm{~A}$ \\
2934.545 & 53.0 & -219.4 & $\mathrm{~A}$ \\
6681.572 & -53.9 & -47.0 & $\mathrm{H}$ \\
6685.581 & -88.0 & 3.2 & $\mathrm{H}$ \\
6686.579 & -143.9 & 173.4 & $\mathrm{H}$ \\
6687.672 & 10.9 & -199.1 & $\mathrm{H}$ \\
6688.573 & 72.7 & -233.6 & $\mathrm{H}$ \\
6682.573 & -152.7 & 175.5 & $\mathrm{H}$ \\
6684.571 & 55.7 & -245.0 & $\mathrm{H}$ \\
\hline
\end{tabular}

Notes. The RVs were determined through cross-correlation in the disentangling process. The $\mathrm{A}$ and $\mathrm{H}$ letters in the last column indicate Aurélie and HEROS data, respectively. 
Table B.5. Newly determined RVs of BD $+60^{\circ} 498$.

\begin{tabular}{ccc}
\hline \hline HJD-2 450 000 & $\begin{array}{c}\mathrm{RV} \\
\mathrm{km} \mathrm{s}^{-1}\end{array}$ & $\begin{array}{c}\sigma(\mathrm{RV}) \\
\mathrm{km} \mathrm{s}^{-1}\end{array}$ \\
\hline 6693.578 & -114.0 & 19.9 \\
6694.581 & -124.7 & 9.7 \\
6695.586 & -63.7 & 38.8 \\
6697.590 & -58.6 & 11.2 \\
6699.569 & -34.7 & 43.9 \\
6700.585 & -11.2 & 9.6 \\
6713.594 & -23.7 & 13.2 \\
6716.596 & -40.4 & 9.9 \\
6960.821 & -47.9 & 24.3 \\
6961.758 & -48.7 & 28.0 \\
6979.719 & -42.9 & 12.4 \\
6980.733 & -69.4 & 13.2 \\
\hline
\end{tabular}

Notes. The RVs are the mean of the values measured on the He I $\lambda \lambda 4026,4121,4144,4388,4471,4713, \mathrm{H} \gamma, \mathrm{H} \beta$ and $\mathrm{He}$ II $\lambda 4686$ lines.

Table B.6. Newly determined RVs of BD+60 499.

\begin{tabular}{ccc}
\hline \hline HJD-2 450 000 & $\begin{array}{c}\mathrm{RV} \\
\mathrm{km} \mathrm{s}^{-1}\end{array}$ & $\begin{array}{c}\sigma(\mathrm{RV}) \\
\mathrm{km} \mathrm{s}^{-1}\end{array}$ \\
\hline 6696.589 & -45.9 & 5.2 \\
6699.628 & -47.3 & 4.9 \\
\hline
\end{tabular}

Notes. The RVs are the mean of the values measured on the He I $\lambda \lambda 4026,4121,4144,4388,4471,4713, \mathrm{H} \gamma, \mathrm{H} \beta$ and He II $\lambda 4686$ lines.

Table B.7. Newly determined RV of BD $+60^{\circ} 513$.

\begin{tabular}{ccc}
\hline \hline HJD-2 450000 & $\begin{array}{c}\mathrm{RV} \\
\mathrm{km} \mathrm{s}^{-1}\end{array}$ & $\begin{array}{c}\sigma(\mathrm{RV}) \\
\mathrm{km} \mathrm{s}^{-1}\end{array}$ \\
\hline 6698.600 & -65.7 & 9.7 \\
\hline
\end{tabular}

Notes. The RV is the mean of the values measured on the He I $\lambda 4471$, $\mathrm{He}$ II $\lambda \lambda 4542,4686$ and $\mathrm{H} \beta$ lines. 